Bull. Soc. math. France

132 (1), 2004, p. 55-80

\title{
DUAL BLOBS AND PLANCHEREL FORMULAS
}

\author{
BY Ju-LEE KIM
}

\begin{abstract}
Let $k$ be a $p$-adic field. Let $G$ be the group of $k$-rational points of a connected reductive group $\mathrm{G}$ defined over $k$, and let $\mathfrak{g}$ be its Lie algebra. Under certain hypotheses on $\mathrm{G}$ and $k$, we quantify the tempered dual $\widehat{G}$ of $G$ via the Plancherel formula on $\mathfrak{g}$, using some character expansions. This involves matching spectral decomposition factors of the Plancherel formulas on $\mathfrak{g}$ and $G$. As a consequence, we prove that any tempered representation contains a good minimal K-type; we extend this result to irreducible admissible representations.

RÉsumé (Blobs duaux et formule de Plancherel). - Soient $k$ un corps $p$-adique, G un groupe réductif connexe défini sur $k, G$ son groupe de points $k$-rationnels et $\mathfrak{g}$ l'algèbre de Lie de G. Sous certaines hypothèses, nous quantifions le dual tempéré $\widehat{G}$ de $G$ par la formule de Plancherel sur $\mathfrak{g}$, en utilisant des développements en caractères. Pour cela, il faut en particulier mettre en correspondance les facteurs de la décomposition spectrale de la formule de Plancherel sur $\mathfrak{g}$ et sur $G$. Comme conséquence, nous démontrons que toute représentation tempérée contient un bon K-type minimal; nous étendons aussi ce résultat aux représentations admissibles irréductibles.
\end{abstract}

Texte reçu le 15 mars 2002, révisé les 22 novembre 2002 et 7 février 2003, accepté le 28 février 2003.

Ju-LeE KIM, School of Mathematics, Institute for Advanced Study, Princeton, NJ 08540 Department of Mathematics Statistics and Computer Science, University of Illinois at Chicago, Chicago, IL 60607 • E-mail : julee@math.uic.edu

2000 Mathematics Subject Classification. - 22E50, 22E35, 20G25.

Key words and phrases. - Representations, $p$-adic groups, Plancherel formula, character expansions.

Research partially supported by NSF grants DMS 9970454 and 9729992. 


\section{Introduction}

Let $k$ be a $p$-adic field, $\mathrm{G}$ a connected reductive group defined over $k$, and $G$ the group of $k$-rational points of G. Let $\widetilde{G}$ be the set of the equivalence classes of irreducible admissible representations of $G$. To study $\widetilde{G}$, representations of compact open subgroups of $G$ have been useful. In particular, Moy and Prasad studied open compact subgroups coming from the theory of the BruhatTits building $\mathcal{B}(G)$ of $G$. They introduced (unrefined) minimal K-types of the form $\mathfrak{s}:=\left(G_{x, \varrho}, \chi\right)$, where $G_{x, \varrho}$ is an open compact subgroup of $G$ associated to $(x, \varrho) \in \mathcal{B}(G) \times \mathbb{R}_{\geq 0}$, and $\chi$ is an irreducible representation of $G_{x, \varrho}$ of a certain type. If we introduce weak associativity, an equivalence relation of minimal K-types (see Definition 2.2.1), we can partition $\widetilde{G}$ as follows

$$
\widetilde{G}=\bigcup_{\mathfrak{s} \in \mathfrak{S}_{\mathrm{K}}}^{\circ} \widetilde{G}_{\mathfrak{s}}
$$

where $\widetilde{G}_{\mathfrak{s}}$ is the set of $\left(\pi, V_{\pi}\right) \in \widetilde{G}$ containing a minimal K-type weakly associated to $\mathfrak{s}$, and $\mathfrak{S}_{\mathrm{K}}$ is the set of equivalence classes of weakly associated minimal K-types.

Let $\widehat{G}$ be the tempered dual of $G$. Recall that $\widehat{G}$ is the support of the Plancherel measures in the unitary dual of $G$. Let $\widehat{\mathfrak{g}}$ be the unitary dual of $\mathfrak{g}$. Then $\widehat{\mathfrak{g}}$ is also a tempered dual of $\mathfrak{g}$. In this paper, we "quantify" the Plancherel integral over each $\widehat{G}_{\mathfrak{s}}:=\widehat{G} \cap \widetilde{G}_{\mathfrak{s}}$ in terms of the Plancherel integral over an appropriate $G$-domain in the Lie algebra $\mathfrak{g}$ of $G$, when the residue characteristic of $k$ is large. Based on the Kirillov theory of compact $p$-adic groups [10], such a quantification first appeared in the work of Howe [11]. To start with, we observe that from Plancherel formulas on $\mathfrak{g}$ and $G$, we have

$$
\int_{\widehat{\mathfrak{g}} \simeq \mathfrak{g}} \widehat{f}(X) \mathrm{d} X=f(0)=\int_{\widehat{G}} \Theta_{\pi}(f \circ \log ) \mathrm{d} \pi
$$

for any $f \in C_{c}^{\infty}(\widehat{\mathfrak{g}}) \simeq C_{c}^{\infty}(\mathfrak{g})$ supported in a small neighborhood of 0 (here, we identify $\mathfrak{g}$ and $\widehat{\mathfrak{g}}$ via Pontrjagin duality). Now, we can refine this equality: we find an equality between spectral decomposition factors of each side (see 1.4.5 and 3.2.6) of $(\mathrm{Pl})$, where spectral components of the right hand side are parameterized by certain minimal K-types, and those of the left hand side by some related $G$-domains in $\widehat{\mathfrak{g}}$.

To be more precise, from now on, we fix a good minimal K-type $\mathfrak{s}=\left(G_{x, \varrho}, \chi\right)$. Note that the depth of $\mathfrak{s}$ is $\varrho$. A minimal K-type is good when its dual blob the dual coset realizing $\chi$ ) is good (see [3] or 1.2.2, 3.2.1). Let $\mathcal{S}$ be the dual blob of $\mathfrak{s}$, and let $\widetilde{G}_{\mathcal{S}} \subset \widetilde{G}_{\mathfrak{s}}$ be the set of $\left(\pi, V_{\pi}\right) \in \widetilde{G}_{\mathfrak{s}}$ containing good K-types weakly associated to $\mathcal{S}$ (in this paper, we introduce three weak associativities: between minimal K-types (2.2.1), between good cosets (1.4.1), between good K-types and good cosets (3.2.3)). Then we match the Plancherel integral over $\widehat{G}_{\mathcal{S}}:=\widehat{G} \cap \widetilde{G}_{\mathcal{S}}$

TOME $132-2004-\mathrm{N}^{\mathrm{O}} 1$ 
with the Fourier transform of some distribution supported on a $G$-domain $\mathfrak{g}_{\mathcal{S}}$ in $\mathfrak{g}$ coming from $\mathcal{S}$. Roughly speaking, $\mathfrak{g}_{\mathcal{S}}$ is the $G$-orbit of good dual blobs weakly associated to $\mathfrak{s}$ (see 1.4.3). In particular, for two good minimal K-types $\mathfrak{s}^{\prime}$ and $\mathfrak{s}^{\prime \prime}$ with dual blobs $\mathcal{S}^{\prime}$ and $\mathcal{S}^{\prime \prime}$, if $\widetilde{G}_{\mathfrak{s}^{\prime}} \cap \widetilde{G}_{\mathfrak{s}^{\prime \prime}}=\varnothing$, then $\mathfrak{g}_{\mathcal{S}^{\prime}} \cap \mathfrak{g}_{\mathcal{S}^{\prime \prime}}=\varnothing$. Moreover, $\mathfrak{g}=\stackrel{\circ}{\cup} \mathfrak{g}_{\mathcal{S}^{\prime}}$ (see 1.4.5) where $\mathcal{S}^{\prime}$ runs over weakly associated classes of good cosets. We remark that the depth of any $\left(\pi, V_{\pi}\right) \in \widetilde{G}_{\mathcal{S}}$ is the same as the depth $\varrho$ of $\mathfrak{s}$, and the depth of $\mathfrak{g}_{\mathcal{S}}$ (see Definition 1.2.3) is $-\varrho$. Let $\mathfrak{g}_{\varrho}:=\bigcup_{x \in \mathcal{B}(G)} \mathfrak{g}_{x, \varrho}$. When $\varrho>0$, we prove that for any $f \in C_{c}^{\infty}\left(\mathfrak{g}_{\varrho}\right)$,

$$
\int_{\mathfrak{g}_{\mathcal{S}}} \widehat{f}(X) \mathrm{d} X=\int_{\widehat{G}_{\mathcal{S}}} \Theta_{\pi}(f \circ \log ) \mathrm{d} \pi .
$$

Summing over $\mathcal{S}$ in weakly associated classes of good cosets, we see

$$
\begin{aligned}
\int_{\mathfrak{g}} \widehat{f}(X) \mathrm{d} X & =\sum_{\mathcal{S}} \int_{\mathfrak{g}_{\mathcal{S}}} \widehat{f}(X) \mathrm{d} X \\
& =\sum_{\mathcal{S}} \int_{\widehat{G}_{\mathcal{S}}} \Theta_{\pi}(f \circ \log ) \mathrm{d} \pi=\int_{\widehat{G}} \Theta_{\pi}(f \circ \log ) \mathrm{d} \pi
\end{aligned}
$$

which leads to the proof that every tempered representation contains a good minimal K-type (see Theorem 4.5.1) when the residue characteristic is large. Under the same hypothesis, we also show that any irreducible admissible representation contains a good minimal K-type. This fact has been already proved (see $[13,2.4 .10]$ ) using more tools from the theory of buildings. Although we still use such tools quite a bit, this work is just an initial step to approach the problem on the exhaustion of the types constructed in [12]. In this sense, the purpose of this paper is rather a piecewise quantification, i.e., a matching of spectral decomposition factors as in (1) (see Theorem 3.3.1). However, one can hope that this analytic approach might be more fruitful for generalization.

To prove the equality in (1), we regard both sides of (1) as distributions on $C_{c}^{\infty}\left(\mathfrak{g}_{\varrho}\right)$. Here, the domain where $(1)$ holds is restricted to $\mathfrak{g}_{\varrho}$, however, this is large enough to single out $\widetilde{G}_{\mathfrak{s}}$ from $\widetilde{G}$ in the following sense: there are $f \in C_{c}^{\infty}\left(\mathfrak{g}_{\varrho}\right)$ such that $\Theta_{\pi}(f \circ \log ) \neq 0$ implies $\pi \in \widetilde{G}_{\mathfrak{s}}$. On the other hand, $C_{c}^{\infty}\left(\mathfrak{g}_{\varrho^{+}}\right)$(here, $\left.\mathfrak{g}_{\varrho^{+}}=\bigcup_{s>\varrho} \mathfrak{g}_{s}\right)$ can not isolate better than the set of depth $\varrho$ representations which strictly contains $\widetilde{G}_{\mathfrak{s}}$. In the case of depth zero representations, it is not possible to distinguish a single class of K-types by dual cosets. Hence replacing $\mathfrak{g}_{\mathcal{S}}$ and $\widetilde{G}_{\mathcal{S}}$ by a $G$-domain $\mathfrak{g}_{0}$ and the set of all depth zero representations $\widetilde{G}_{0}$, we prove an analogous equality on $C_{c}^{\infty}\left(\mathfrak{g}_{0^{+}}\right)$. However, this is good enough to prove that any tempered representation contains a good minimal K-type.

We approach this problem via various character expansions and homogeneity results. When $\varrho=0$, by the work of Waldspurger and DeBacker (see [18], [5]), we know that the Harish-Chandra-Howe local character expansion is valid on 
the set $\mathfrak{g}_{0+}$ of topologically nilpotent elements in $\mathfrak{g}$. We show that the distributions in (1) are in the span of Fourier transforms of nilpotent orbital integrals when restricted to $\mathfrak{g}_{0^{+}}$. Then we match two distributions using Gelfand-Graev functions as test functions as in [4].

If $\varrho>0$, the Harish-Chandra-Howe expansions are not enough, because the set $\mathfrak{g}_{\varrho^{+}}$where they hold is not big enough. Hence we use $\Gamma$-asymptotic expansions of representations in $\widetilde{G}_{\mathcal{S}}$. In [13], F. Murnaghan and the author proved that such expansions are valid on the $G$-domain $\mathfrak{g}_{\varrho}$. More precisely, assume that $\pi$ contains a good type coming from a good element $\Gamma$ of depth $-\varrho$ (see [3] or 1.2.1-1.2.2 for definition). Denote the set of $G$-orbits whose closure contains $\Gamma$ by $\mathcal{O}(\Gamma)$. Then we can express the character distribution $\Theta_{\pi}$ as a linear combination of Fourier transforms of orbital integrals $\mu_{\mathcal{O}}$ with $\mathcal{O} \in \mathcal{O}(\Gamma)$. That is, if $\pi$ is an irreducible admissible representation of $G$ containing $\left(G_{x, \varrho}, \chi\right)$, and if $\chi$ is realized by a good element $\Gamma$, we prove that there are $c_{\mathcal{O}}(\pi) \in \mathbb{C}$ indexed by $\mathcal{O}(\Gamma)$ such that

$$
\Theta_{\pi}(\exp X)=\sum_{\mathcal{O} \in \mathcal{O}(\Gamma)} c_{\mathcal{O}}(\pi) \widehat{\mu_{\mathcal{O}}}(X)
$$

and this expansion is valid on $\mathfrak{g}_{\varrho} \cap \mathfrak{g}_{\text {reg }}$. Then we show that the distributions in (1) are in the span of Fourier transforms of $\mu_{\mathcal{O}}$ with $\mathcal{O} \in \mathcal{O}(\Gamma)$ when restricted to $\mathfrak{g}_{\varrho}$, and we match two distributions using some test functions found in [13].

In the first section, we recall some basic definitions related to this work, and state the hypotheses that we use at various places of this paper. We also define the weak associativity of good cosets, which induces a partition of $\mathfrak{g}$ accordingly $(\S 1.4)$. Then we show that this partition induces a spectral decomposition as in (2) (see Lemma 1.4.5). In Section 2, we define the weak associativity of minimal K-types, which induces a partition of $\widetilde{G}$. In Section 3 , we relate the partitions on $\mathfrak{g}$ and $\widetilde{G}$ found in the first two sections. Section 4 is basically devoted to proving the equality in (1) (see Theorem 3.3.1). As an application, in the end of Section 4, we prove that any tempered representation contains a good minimal K-type; we also discuss the extension of this result to irreducible admissible representations. As a corollary, we also get a new spectral decomposition of the delta distribution on $G$ where each decomposition factor is parameterized by the $G$-orbit of a good dual blob.

Acknowledgments. - This work was motivated from conversations with R. Howe. I would like to thank him for helpful discussions. I would like to thank A.-M. Aubert, S. DeBacker and F. Murnaghan for helpful comments. I thank the Institute for Advanced Study for its friendly and stimulating atmosphere.

Notation and Conventions. - Let $k$ be a $p$-adic field (a finite extension of $\mathbb{Q}_{p}$ ) with residue field $\mathbb{F}_{p^{n}}$. Let $\nu=\nu_{k}$ be the valuation on $k$ such that

TOME $132-2004-\mathrm{N}^{\mathrm{O}} 1$ 
$\nu\left(k^{\times}\right)=\mathbb{Z}$. Let $\bar{k}$ be an algebraic closure of $k$. For an extension field $E$ of $k$, let $\nu_{E}$ be the valuation on $E$ extending $\nu$. We will just write $\nu$ for $\nu_{E}$. Let $\mathcal{O}_{E}$ be the ring of integers of $E$ with prime ideal $\mathfrak{p}_{E}$. Let $\Lambda$ be a fixed additive character of $k$ such that $\Lambda_{\mid \mathcal{O}_{k}} \neq 1$ and $\Lambda_{\mathfrak{|}_{k}}=1$.

Let $\mathrm{G}$ be a connected reductive group defined over $k$, and $\mathrm{G}(E)$ the group of $E$-rational points of $\mathrm{G}$. We denote by $G$ the group of $k$-rational points of $\mathrm{G}$. Denote the Lie algebras of $G$ and $G(E)$ by $\mathfrak{g}$ and $\mathfrak{g}(E)$, respectively. Denote by $\mathfrak{g}^{*}$ and $\mathfrak{g}^{*}(E)$ the linear duals of $\mathfrak{g}$ and $\mathfrak{g}(E)$ respectively. We write $\mathfrak{g}$ and $\mathfrak{g}^{*}$ for the vector space of $k$-rational points of $\mathfrak{g}$ and $\mathfrak{g}^{*}$ respectively. In general, we use bold characters $\mathrm{H}, \mathrm{M}, \mathrm{N}$, etc. to denote algebraic groups defined over $k$, corresponding Roman characters $H, M$ and $N$ to denote the groups of $k$-points, and $\mathfrak{h}, \mathfrak{m}$ and $\mathfrak{n}$ to denote the Lie algebras of $H, M$ and $N$.

Denote the set of regular elements in $\mathfrak{g}$ by $\mathfrak{g}_{\text {reg. }}$. Let $\mathcal{N}$ be the set of nilpotent elements in $\mathfrak{g}$. There are different notions of nilpotency. However, since we assume that $\operatorname{char}(k)=0$, those notions are all the same. We refer to [14], [6] for more discussion of this.

If $X$ is a topological space with a Borel measure $\mathrm{d} x$ and if $Y$ is a subset of $X, \operatorname{vol}_{X}(Y)$ denotes the volume of $Y$ with respect to $\mathrm{d} x$.

For any subset $S$ in $\mathfrak{g}$, we denote by $[S]$ the characteristic function of $S$, and by $-S$ the set $\{-s \mid s \in S\}$. For $g \in G,{ }^{g} Z$ denotes $g Z g^{-1}$ and for $H \subset G,{ }^{H} S$ denotes $\left\{{ }^{g} Z \mid Z \in S, g \in H\right\}$.

Let $\widetilde{G}$ be the set of equivalence classes of irreducible admissible representations of $G$. Let $\widehat{G}$ be the subset of $\widetilde{G}$ which consists of equivalence classes of tempered representations of $G$.

\section{Good cosets and $\mathfrak{g}$}

1.1. Moy-Prasad filtrations. - For a finite extension $E$ of $k$, let $\mathcal{B}(\mathrm{G}, E)$ denote the extended Bruhat-Tits building of $G$ over $E$. For a maximal torus $T$ in $\mathrm{G}$, if it splits over $E$, let $\mathcal{A}(\mathrm{T}, E)$ be the corresponding apartment over $E$. It is known that if $E^{\prime}$ is a tamely ramified Galois extension of $E, \mathcal{B}(\mathrm{G}, E)$ can be embedded into $\mathcal{B}\left(\mathrm{G}, E^{\prime}\right)$ and its image is equal to the set of the Galois fixed points in $\mathcal{B}\left(\mathrm{G}, E^{\prime}\right)$ (see $[17,5.11]$ or $[16]$ ). Moreover, we have

$$
\mathcal{A}(\mathrm{T}, E)=\mathcal{A}\left(\mathrm{T}, E^{\prime}\right) \cap \mathcal{B}(\mathrm{G}, E) .
$$

We let $\mathcal{A}(\mathrm{T}, k):=\mathcal{A}(\mathrm{T}, E) \cap \mathcal{B}(\mathrm{G}, k)$.

Regarding $\mathrm{G}$ as a group defined over $E$, Moy and Prasad associate $\mathfrak{g}(E)_{x, r}$ and $\mathrm{G}(E)_{x,|r|}$ to $(x, r) \in \mathcal{B}(\mathrm{G}, E) \times \mathbb{R}$ with respect to the valuation normalized as follows [14]: let $E^{u}$ be the maximal unramified extension of $E$, and $L$ the minimal extension of $E^{u}$ over which $G$ splits. Then the valuation used by Moy and Prasad maps $L^{\times}$onto $\mathbb{Z}$. 
In a similar way, with respect to our normalized valuation $\nu$, we can define filtrations in $\mathfrak{g}(E)$ and $\mathrm{G}(E)$. Then our $\mathfrak{g}(E)_{x, r}$ and $\mathrm{G}(E)_{x, r}$ correspond to $\mathfrak{g}(E)_{x, e \ell r}$ and $\mathrm{G}(E)_{x, e \ell r}$ of Moy and Prasad, where $e=e(E / k)$ is the ramification index of $E$ over $k$ and $\ell=\left[L: E^{u}\right]$. Hence, if $\varpi_{E}$ is a uniformizing element of $E$, our filtrations satisfy $\varpi_{E} \mathfrak{g}(E)_{x, r}=\mathfrak{g}(E)_{x, r+1 / e}$ while theirs satisfy $\varpi_{E} \mathfrak{g}(E)_{x, r}=\mathfrak{g}(E)_{x, r+\ell}$.

This normalization is chosen to have the following property $[1,1.4 .1]$ : for a tamely ramified Galois extension $E^{\prime}$ of $E$ and $x \in \mathcal{B}(\mathrm{G}, E) \subset \mathcal{B}\left(\mathrm{G}, E^{\prime}\right)$, we have

$$
\mathfrak{g}(E)_{x, r}=\mathfrak{g}\left(E^{\prime}\right)_{x, r} \cap \mathfrak{g}(E) .
$$

If $r>0$, we also have

$$
\mathrm{G}(E)_{x, r}=\mathrm{G}\left(E^{\prime}\right)_{x, r} \cap \mathrm{G}(E) .
$$

REMARK 1.1.1. - Let $r \in \frac{1}{e} \mathbb{Z}$. Two points $x$ and $y$ in $\mathcal{B}(\mathrm{G}, E)$ lie in the same facet if and only if

$$
\mathfrak{g}(E)_{x, r}=\mathfrak{g}(E)_{y, r} \quad \text { and } \quad \mathfrak{g}(E)_{x, r^{+}}=\mathfrak{g}(E)_{y, r^{+}} .
$$

1.1.2. - For simplicity, we put $\mathcal{B}(G):=\mathcal{B}(\mathrm{G}, k)$ and $\mathfrak{g}_{x, r}:=\mathfrak{g}(k)_{x, r}$, etc. We will also use the following notation. Let $r \in \mathbb{R}$. Then,

1) $\mathfrak{g}_{x, r^{+}}=\bigcup_{s>r} \mathfrak{g}_{x, s}$ and $G_{x,|r|^{+}}=\bigcup_{s>|r|} G_{x, s}, \quad x \in \mathcal{B}(G)$;

2) $\mathfrak{g}_{x, r}^{*}=\left\{\chi \in \mathfrak{g}^{*} \mid \chi\left(\mathfrak{g}_{x,(-r)^{+}}\right) \subset \mathfrak{p}_{k}\right\}, \quad x \in \mathcal{B}(G)$;

3) $\mathfrak{g}_{r}=\bigcup_{x \in \mathcal{B}(G)} \mathfrak{g}_{x, r}$ and $\mathfrak{g}_{r+}=\bigcup_{s>r} \mathfrak{g}_{s}$;

4) $G_{r}=\bigcup_{x \in \mathcal{B}(G)} G_{x, r}$ and $G_{r+}=\bigcup_{s>r} G_{s}$ for $r \geq 0$.

For $(x, r) \in \mathcal{B}(\mathrm{G}, E) \times \mathbb{R}$, we can define corresponding objects in $\mathfrak{g}(E), \mathfrak{g}^{*}(E)$ and $\mathrm{G}(E)$. We will denote them using $(E)$.

1.2. Depth functions and good elements. - Recall that the depth function on $\mathcal{B}(\mathrm{G}, k) \times \mathfrak{g}$ is a function $\mathrm{d}: \mathcal{B}(\mathrm{G}, k) \times \mathfrak{g} \rightarrow \mathbb{R}$ defined as follows: for $X \in \mathfrak{g}$ and $x \in \mathcal{B}(\mathrm{G}, k)$, let $\mathrm{d}(x, X)=r$ be the depth of $X$ in the $x$-filtration, that is, $r$ is the unique real number such that $X \in \mathfrak{g}_{x, r} \backslash \mathfrak{g}_{x, r^{+}}$. We also define

$$
\mathrm{d}(X)=\sup _{x \in \mathcal{B}(\mathrm{G}, k)} \mathrm{d}(x, X) .
$$

Note that the depth $\mathrm{d}(X)$ of $X$ is the unique $r$ in $\mathbb{R} \cup\{\infty\}$ such that $X \in \mathfrak{g}_{r} \backslash \mathfrak{g}_{r^{+}}$. Moreover, $\mathrm{d}$ is well defined and locally constant on $\mathfrak{g} \backslash \mathcal{N}$, and it is $\infty$ on $\mathcal{N}$ (see $[2,3.3 .4]$ ). We can also define a depth function $\mathrm{d}^{E}$ over a finite extension $E$ of $k$. If $E$ is tamely ramified, thanks to our normalization of valuation, we observe that for any $x \in \mathcal{B}(\mathrm{G}, k)$ and $X \in \mathfrak{g}, \mathrm{d}(x, X)=\mathrm{d}^{E}(x, X)$ and $\mathrm{d}(X)=\mathrm{d}^{E}(X)$ (see [2]). Hence we may omit the superscript ' $E$ ' in that case.

TOME $132-2004-\mathrm{N}^{\mathrm{O}} 1$ 
Let $\mathrm{T}$ be a maximal $k$-torus which splits over a tamely ramified Galois extension $E$ of $k$, and let $\mathfrak{t}$ be its Lie algebra. Then $T:=\mathrm{T}(k)$ and $\mathfrak{t}:=\mathfrak{t}(k)$ have the following filtrations:

- for $r \in \mathbb{R}, \quad \mathfrak{t}_{r}:=\left\{\Gamma \in \mathfrak{t} \mid \nu(d \chi(\Gamma)) \geq r\right.$ for all $\left.\chi \in \mathbf{X}^{*}(\mathrm{~T})\right\}$ and

- for $r>0, T_{r}:=\left\{t \in T \mid \nu(\chi(t)-1) \geq r\right.$ for all $\left.\chi \in \mathrm{X}^{*}(\mathrm{~T})\right\}$.

Note that $T_{r}=T \cap \mathrm{T}(E)_{r}$ and $\mathfrak{t}_{r}=\mathfrak{t} \cap \mathfrak{t}(E)_{r}$.

DeFinition 1.2.1 (see [3]). - Let T be a maximal $k$-torus which splits over a tamely ramified Galois extension of $k$, and let $\mathfrak{t}$ be its Lie algebra.

1) If $\Gamma \in \mathfrak{t}_{r} \backslash \mathfrak{t}_{r^{+}}$, we say that $\Gamma$ is of depth $r$ with respect to $\mathrm{T}$, and we write $\mathrm{d}_{\mathrm{T}}(\Gamma)=r$.

2) Let $\Gamma \in \mathfrak{g}$ be a semisimple element of depth $r$ with respect to $t$. Then $\Gamma$ is called good with respect to $\mathrm{T}$ if for every root $\alpha$ of $\mathrm{G}$ with respect to $\mathrm{T}, d \alpha(\Gamma)$ is either zero or has valuation $r$.

The depth and goodness of semisimple elements do not depend on the choice of $\mathrm{T}$, and in fact $\mathrm{d}_{\mathrm{T}}(\Gamma)=\mathrm{d}(\Gamma)$ (see $\left.[13,2.1 .2],[1]\right)$. Note that $0 \in \mathfrak{g}$ is a good element of depth $\infty$.

Definition 1.2 .2 (see [3], [13]). - 1) Let $r<0$. A coset $\mathcal{S}=X+\mathfrak{g}_{x, r^{+}}$ with $X \in \mathfrak{g}_{x, r} \backslash \mathfrak{g}_{x, r^{+}}$is good if there is a good element $\Gamma$ of depth $r$ such that $\Gamma+\mathfrak{g}_{x, r^{+}}=X+\mathfrak{g}_{x, r^{+}}$and $x \in \mathcal{B}\left(\mathrm{C}_{\mathrm{G}}(\Gamma), k\right)$.

2) For $x \in \mathcal{B}(\mathrm{G}, k), \mathcal{S}:=\mathfrak{g}_{x, 0}$ is called a 0 -good coset.

DeFinition AND REMARK 1.2.3. - Let $\mathcal{S}$ be a good coset. Define the $\operatorname{depth} \mathrm{d}(\mathcal{S})$ of $\mathcal{S}$ as

$$
\mathrm{d}(\mathcal{S}):=\min _{X \in \mathcal{S}} \mathrm{d}(X) .
$$

Then if $\mathcal{S}$ is a 0 -good coset, $\mathrm{d}(\mathcal{S})=0$. If $\mathcal{S}=\Gamma+\mathfrak{g}_{x, r^{+}}$with $r<0$, for any $X \in \mathcal{S}$, then $\mathrm{d}(\mathcal{S})=\mathrm{d}(X)=r$ by $[2,3.3 .7]$.

1.3. $\mathcal{B}\left(\mathbf{G}^{\prime}, \boldsymbol{k}\right)$. - Let $\mathrm{T}$ be a maximal $k$-torus in $\mathrm{G}$ which splits over a tamely ramified Galois extension $E$ of $k$, and let $\mathfrak{t}$ be its Lie algebra. Let $\Gamma$ be a semisimple element in $\mathfrak{t}$. Let $G^{\prime}$ denote the centralizer $C_{G}(\Gamma)$ of $\Gamma$ in $G$, and $\mathfrak{g}^{\prime}$ its Lie algebra. Then $\left(G^{\prime}, G\right)$ forms a twisted Levi sequence, that is, $G^{\prime}(E)$ is an $E$-Levi subgroup of $\mathrm{G}(E)$ (see [20]). In particular, if $\Gamma$ splits over $k, G^{\prime}$ is a Levi subgroup of $G$. In general, $\mathrm{G}^{\prime}(E)$ is a Levi subgroup of $\mathrm{G}(E)$, and hence there is a Galois equivariant embedding of $\mathcal{B}\left(\mathrm{G}^{\prime}, E\right)$ into $\mathcal{B}(\mathrm{G}, E)$, which in turn induces an embedding of $\mathcal{B}\left(\mathrm{G}^{\prime}, k\right)$ into $\mathcal{B}(\mathrm{G}, k)$ (see $[1, \S 1.9]$ or $[20,2.11]$ ). Such embeddings are unique modulo translation by elements of $\mathbf{X}_{*}\left(\mathbf{Z}_{\mathrm{G}^{\prime}}, k\right) \otimes \mathbb{R}$. However, the images remain the same.

Fix such an embedding $i: \mathcal{B}\left(\mathrm{G}^{\prime}, k\right) \rightarrow \mathcal{B}(\mathrm{G}, k)$. Then we will regard $\mathcal{B}\left(\mathrm{G}^{\prime}, k\right)$ as a subset of $\mathcal{B}(\mathrm{G}, k)$ and write just $x$ for $i(x)$. For any $x \in \mathcal{B}\left(\mathrm{G}^{\prime}, k\right)$, the 
associated filtrations on $G^{\prime}:=\mathrm{G}^{\prime}(k)$ and $\mathfrak{g}^{\prime}:=\mathfrak{g}^{\prime}(k)$ are given as follows (see $[1,1.9 .1])$ :

$$
\begin{aligned}
& G_{x, r}^{\prime}=\mathrm{G}(E)_{x, r} \cap G^{\prime}=G_{x, r} \cap G^{\prime} \text { for } r>0, \\
& \mathfrak{g}_{x, r}^{\prime}=\mathfrak{g}(E)_{x, r} \cap \mathfrak{g}^{\prime}=\mathfrak{g}_{x, r} \cap \mathfrak{g}^{\prime} \quad \text { for any } r \in \mathbb{R} .
\end{aligned}
$$

For later use, we need some lemmas on good cosets. The implication of Lemma 1.3.2 on good K-types can be found in Corollary 3.2.2. We first need to recall the following. Let $r \in \mathbb{R}$. In [6], DeBacker defined the notion of generalized $r$-facets in the affine building of a reductive group. For the purposes of this paper, it suffices to consider the set of generalized $r$-facets in $\mathcal{B}\left(\mathrm{G}^{\prime}, k\right)$.

Definition 1.3.1 (see [6]). - For $x \in \mathcal{B}\left(\mathrm{G}^{\prime}, k\right)$, define

$$
\begin{aligned}
F^{*}(x): & =\left\{y \in \mathcal{B}\left(\mathrm{G}^{\prime}, k\right) \mid \mathfrak{g}_{x, r}^{\prime}=\mathfrak{g}_{y, r}^{\prime} \text { and } \mathfrak{g}_{x, r^{+}}^{\prime}=\mathfrak{g}_{y, r^{+}}^{\prime}\right\} \\
& =\left\{y \in \mathcal{B}\left(\mathrm{G}^{\prime}, k\right) \mid G_{x,|r|}^{\prime}=G_{y,|r|}^{\prime} \text { and } G_{x,|r|^{+}}^{\prime}=G_{y,|r|^{+}}^{\prime}\right\}, \\
\mathcal{F}(r): & =\left\{F^{*}(x) \mid x \in \mathcal{B}\left(\mathrm{G}^{\prime}, k\right)\right\} .
\end{aligned}
$$

An element in $\mathcal{F}(r)$ is called a generalized $r$-facet in $\mathcal{B}\left(\mathrm{G}^{\prime}, k\right)$. For $F^{*}:=F^{*}(x)$ in $\mathcal{F}(r)$, define

$$
\mathfrak{g}_{F^{*}}^{\prime}:=\mathfrak{g}_{x, r}^{\prime}, \quad \mathfrak{g}_{F^{*}}^{+}:=\mathfrak{g}_{x, r^{+}}^{\prime}, \quad G_{F^{*}}^{\prime}:=G_{x, r}^{\prime}, \quad G_{F^{*}}^{+}:=G_{x, r^{+}}^{\prime} .
$$

Lemma 1.3.2. - Let $x \in \mathcal{B}\left(\mathrm{G}^{\prime}, k\right)$, and let $X^{\prime} \in \mathfrak{g}_{x, r} \cap \mathfrak{g}_{r^{+}}^{\prime}$. Then there exist $g \in G_{x, 0}^{\prime}$ and $y \in \mathcal{B}\left(\mathrm{G}^{\prime}, k\right)$ such that

(i) $\Gamma+{ }^{g} X^{\prime}+\mathfrak{g}_{x, r^{+}} \subset \Gamma+\mathfrak{g}_{y, r^{+}}$, and

(ii) $\mathfrak{g}_{x, r^{+}} \subset \mathfrak{g}_{y, r^{+}}$and $\mathfrak{g}_{x, r} \supset \mathfrak{g}_{y, r}$.

Proof. - Since $X^{\prime} \in \mathfrak{g}_{r^{+}}^{\prime}$, there is a nilpotent element $n^{\prime} \in \mathfrak{g}^{\prime}$ such that

$$
\Gamma+X^{\prime}+\mathfrak{g}_{x, r^{+}}^{\prime}=\Gamma+n^{\prime}+\mathfrak{g}_{x, r^{+}}^{\prime} .
$$

Let $F^{*} \subset \mathcal{B}\left(\mathrm{G}^{\prime}, k\right)$ be a maximal generalized $r$-facet which contains $x$ in the closure. From $[15,6.3]$, we can deduce that there is a $g \in G_{x, 0}^{\prime}$ such that $n^{\prime \prime}={ }^{g} n^{\prime} \in \mathfrak{g}_{F^{*}}^{+}$. Then

$$
\Gamma+{ }^{g} X^{\prime}+\mathfrak{g}_{x, r^{+}}=\Gamma+{ }^{g} n^{\prime}+\mathfrak{g}_{x, r^{+}}=\Gamma+n^{\prime \prime}+\mathfrak{g}_{x, r^{+}} .
$$

Recall that $\Gamma$ splits over a tamely ramified Galois extension $E$ of $k$. Hence $\mathrm{G}^{\prime}(E)$ is an $E$-Levi subgroup of $\mathrm{G}(E)$. Let $\mathrm{T}$ be a maximal $E$-split torus in $\mathrm{G}$ such that $\Gamma \in \mathfrak{t}(E), F^{*} \cap \mathcal{A}(\mathrm{T}, E) \neq \varnothing$, and $x \in \mathcal{A}(\mathrm{T}, E)$. Note that since $\mathrm{T} \subset \mathrm{G}^{\prime}, \mathcal{A}(\mathrm{T}, E) \subset \mathcal{B}\left(\mathrm{G}^{\prime}, E\right)$. Let $C \subset \mathcal{A}(\mathrm{T}, E)$ be a chamber in $\mathcal{B}(\mathrm{G}, E)$ such that $x \in \bar{C}$ and $F^{*} \cap \bar{C} \neq \varnothing$. Choose $\tilde{y} \in C$. Then

$$
\mathfrak{g}(E)_{\tilde{y}, r} \subset \mathfrak{g}(E)_{x, r} \quad \text { and } \quad \mathfrak{g}(E)_{x, r^{+}} \subset \mathfrak{g}(E)_{\tilde{y}, r^{+}} .
$$

TOME $132-2004-\mathrm{N}^{\mathrm{O}} 1$ 
For any $z \in F^{*} \cap \bar{C}, \mathfrak{g}_{z, r^{+}}^{\prime}=\mathfrak{g}_{F^{*}}^{\prime+}$ and $\mathfrak{g}^{\prime}(E)_{z, r^{+}} \subset \mathfrak{g}^{\prime}(E)_{\tilde{y}, r^{+}}$. Hence $n^{\prime \prime}$ is an element of $\mathfrak{g}_{F^{*}}^{\prime+} \subset \mathfrak{g}^{\prime}(E)_{\tilde{y}, r^{+}}$. Let $y$ be the center of mass of the Galois orbit of $\tilde{y}$. Then $y \in \mathcal{A}(\mathrm{T}, k) \subset \mathcal{B}\left(\mathrm{G}^{\prime}, k\right)$. Note that

$$
\begin{gathered}
n^{\prime \prime} \in \bigcap_{\sigma \in \operatorname{Gal}(E / k)} \mathfrak{g}(E)_{\tilde{y}^{\sigma}, r^{+}} \subset \mathfrak{g}(E)_{y, r^{+}}, \\
\mathfrak{g}(E)_{x, r^{+}} \subset \bigcap_{\sigma \in \operatorname{Gal}(E / k)} \mathfrak{g}(E)_{\tilde{y}^{\sigma}, r^{+}} \subset \mathfrak{g}(E)_{y, r^{+}}, \\
\mathfrak{g}(E)_{y, r} \subset \sum_{\sigma \in \operatorname{Gal}(E / k)} \mathfrak{g}(E)_{\tilde{y}^{\sigma}, r} \subset \mathfrak{g}(E)_{x, r}
\end{gathered}
$$

Hence $y$ satisfies the required properties.

Remarks 1.3.3. - Let $\Gamma$ be a good element and let $\mathrm{G}^{\prime}:=\mathrm{C}_{\mathrm{G}}(\Gamma)$. Let $x$ in $\mathcal{B}\left(\mathrm{G}^{\prime}, k\right)$. We recall some results from $[13, \S 2]$.

1) If $X^{\prime} \in \mathfrak{g}_{x, r} \cap \mathfrak{g}_{r^{+}}^{\prime}$, we have ${ }_{x, 0^{+}}\left(\Gamma+X^{\prime}+\mathfrak{g}_{x, r^{+}}^{\prime}\right)=\Gamma+X^{\prime}+\mathfrak{g}_{x, r^{+}}$.

2) $\mathcal{B}\left(\mathrm{G}^{\prime}, k\right)=\{x \in \mathcal{B}(\mathrm{G}, k) \mid \mathrm{d}(x, \Gamma)=\mathrm{d}(\Gamma)\}$.

3) For any $y \in \mathcal{B}(\mathrm{G}, k) \backslash \mathcal{B}\left(\mathrm{G}^{\prime}, k\right)$ and $X^{\prime} \in \mathfrak{g}_{r^{+}}^{\prime}, \mathrm{d}\left(y, \Gamma+X^{\prime}\right)$ is strictly less than $r$.

4) Let $X_{1}, X_{2} \in \Gamma+\mathfrak{g}_{x, r^{+}}^{\prime}$. If ${ }^{g} X_{1}=X_{2}$ for some $g \in G$, then $g \in C_{G}(\Gamma)$.

Lemma 1.3.4. - Let $\Gamma$ be a good element and let $\mathrm{G}^{\prime}:=\mathrm{C}_{\mathrm{G}}(\Gamma)$. Let $x$ in $\mathcal{B}\left(\mathrm{G}^{\prime}, k\right)$, and let $X^{\prime} \in \mathfrak{g}_{x, r} \cap \mathfrak{g}_{r^{+}}^{\prime}$. Let $Z+\mathfrak{g}_{z, r^{+}}$be a coset in $\mathfrak{g}_{z, r}$ where $z \in \mathcal{B}(\mathrm{G}, k)$. If $\left(\Gamma+X^{\prime}+\mathfrak{g}_{x, r^{+}}\right) \cap\left(Z+\mathfrak{g}_{z, r^{+}}\right) \neq \varnothing$, then there exist $h \in G_{x, 0^{+}}$ and $n^{\prime} \in \mathfrak{g}_{h z, r}^{\prime} \cap \mathcal{N}$ such that $h z \in \mathcal{B}\left(\mathrm{G}^{\prime}, k\right)$ and $Z+\mathfrak{g}_{z, r^{+}}=h^{-1}\left(\Gamma+n^{\prime}\right)+\mathfrak{g}_{z, r^{+}}$.

Proof. - Since $\left(\Gamma+X^{\prime}+\mathfrak{g}_{x, r^{+}}\right) \cap\left(Z+\mathfrak{g}_{z, r^{+}}\right) \neq \varnothing$, by Remark 1.3.3, 1), there exist $h \in G_{x, 0^{+}}$and $Y^{\prime} \in X^{\prime}+\mathfrak{g}_{x, r^{+}}^{\prime}$ such that $h^{-1}\left(\Gamma+Y^{\prime}\right) \in Z+\mathfrak{g}_{z, r^{+}}$. Hence $\Gamma+Y^{\prime}+\mathfrak{g}_{h z, r^{+}}={ }^{h} Z+\mathfrak{g}_{h z, r^{+}}$. From Remark 1.3.3, 3), $h z \in \mathcal{B}\left(\mathrm{G}^{\prime}, k\right)$. Since $Y^{\prime} \in \mathfrak{g}_{r^{+}}^{\prime}$ by $[2,3.2 .2,3.2 .6]$, there is an $n^{\prime} \in \mathfrak{g}_{h z, r}^{\prime} \cap \mathcal{N}$ such that $\Gamma+n^{\prime}+\mathfrak{g}_{h z, r^{+}}=$ $\Gamma+Y^{\prime}+\mathfrak{g}_{h z, r^{+}}$. Hence $Z+\mathfrak{g}_{z, r^{+}}=h^{-1}\left(\Gamma+n^{\prime}\right)+\mathfrak{g}_{z, r^{+}}$.

LEMma 1.3.5. - Let $\mathrm{T}$ be a maximal $k$-torus in $\mathrm{G}$ which splits over a tamely ramified extension $E$. Let $\Gamma_{1}, \Gamma_{2} \in \mathfrak{t}$ be two good semisimple elements of depth $r$ such that $\Gamma_{1} \equiv \Gamma_{2}\left(\bmod \mathfrak{t}_{r^{+}}\right)$. Then $C_{G}\left(\Gamma_{1}\right)=C_{G}\left(\Gamma_{2}\right)$. Moreover, $\Gamma_{1}-\Gamma_{2}$ is in the center of the Lie algebra of $C_{G}\left(\Gamma_{1}\right)$.

Proof. - Let $x \in \mathcal{A}(\mathrm{T}, k)$. Then $\Gamma_{1}+\mathfrak{g}_{x, r^{+}}=\Gamma_{2}+\mathfrak{g}_{x, r^{+}}$is a good coset. Applying Remark 1.3.3,4) for $X_{1}=X_{2}=\Gamma_{1}$ and $\Gamma=\Gamma_{2}$, we have $C_{G}\left(\Gamma_{1}\right) \subset$ $C_{G}\left(\Gamma_{2}\right)$. Similarly, $C_{G}\left(\Gamma_{2}\right) \subset C_{G}\left(\Gamma_{1}\right)$. Hence $C_{G}\left(\Gamma_{1}\right)=C_{G}\left(\Gamma_{2}\right)$. The second statement follows from the first one.

BULletin DE LA SOCiÉtÉ MATHÉMATIQUE DE FRANCE 
Hypotheses 1.3.6. - Here we list the hypotheses used in various places of this paper. These are already used in [4], [5], [13]. We will state explicitly whenever these hypotheses are necessary.

(HB) There is a nondegenerate $G$-invariant symmetric bilinear form B on $\mathfrak{g}$ such that $\mathfrak{g}_{x, r}^{*}$ is identified with $\mathfrak{g}_{x, r}$ via the map $\Omega: \mathfrak{g} \rightarrow \mathfrak{g}^{*}$ defined by $\Omega(X)(Y)=\mathrm{B}(X, Y)$.

(HGT) Every maximal $k$-torus $\mathrm{T}$ in $\mathrm{G}$ splits over a tamely ramified Galois extension, and for any $r \in \mathbb{R}$, any coset in $\mathfrak{t}_{r} / \mathfrak{t}_{r}$ contains a good semisimple element (as defined in $\S 2.1$ ).

$(\mathrm{H} k)$ The residue characteristic $p$ is large enough so that

1) with respect to the adjoint representation $G \rightarrow G L(\mathfrak{g})$, the exponential map (resp. the $\log$ map) is defined on the $G$-domain $\mathfrak{g}_{0^{+}}$of $\mathfrak{g}$ (resp. on $G_{0^{+}}$), and $\exp \left(\mathfrak{g}_{x, r}\right)=G_{x, r}$ (resp. $\log \left(G_{x, r}\right)=\mathfrak{g}_{x, r}$ ) for any $x \in \mathcal{B}(\mathrm{G}, k)$ and $r>0$,

2) the hypotheses in $[5,3.5 .2]$ and $[4,4.4]$ are valid.

Groups satisfying (HB) and (HGT) are discussed in [3]. From Proposition 4.1 and Proposition 5.4 of [3], we see that if $p$ is large enough, (HB) and (HGT) are valid. For $(\mathrm{H} k)$, you can find more precise bounds using the CampbellHausdorff formula (see $[12,3.1 .1,3.2 .3]$ ). Hypothesis (HB) is included in $[5$, 3.5.2]. Hypothesis $(\mathrm{H} k), 2)$ is necessary to apply results of [4] and [5]. We refer to $[5,3.5 .2]$ and $[4,4.4]$ for details.

\subsection{Partition of $\mathfrak{g}$ via good cosets}

Definition 1.4.1. - Let $\mathcal{S}$ and $\mathcal{S}^{\prime}$ be two good cosets in $\mathfrak{g}$. We say that $\mathcal{S}$ is associated to $\mathcal{S}^{\prime}$ if there is $g \in G$ such that $\mathcal{S} \cap \cap^{g} \mathcal{S}^{\prime} \neq \varnothing$. We say that $\mathcal{S}$ is weakly associated to $\mathcal{S}^{\prime}$ and write $\mathcal{S} \sim \mathcal{S}^{\prime}$, if either $\mathcal{S}$ and $\mathcal{S}^{\prime}$ are 0-good cosets, or if $\mathrm{d}(\mathcal{S})=\mathrm{d}\left(\mathcal{S}^{\prime}\right)=r$ and there is a sequence of nondegenerate dual cosets $\mathcal{S}=\mathcal{Y}_{0}, \mathcal{Y}_{1}, \cdots, \mathcal{Y}_{k}=\mathcal{S}^{\prime}$ of the form $\mathcal{Y}_{i}=Y_{i}+\mathfrak{g}_{x_{i}, r}$ and $Y_{i} \in \mathfrak{g}_{x_{i}, r}$, such that $\mathcal{Y}_{i} \cap{ }^{g_{i}} \mathcal{Y}_{i+1} \neq \varnothing$ for some $g_{i} \in G$.

It is straightforward that the above weak associativity is an equivalence relation. We denote by $\mathfrak{S}$ the set of equivalence classes of weakly associated good cosets. The following is a corollary of Lemma 1.3.2 and Lemma 1.3.4. It characterizes weakly associated good cosets.

LEMMA 1.4.2. - Let $\Gamma$ be a good element and $\mathrm{G}^{\prime}=\mathrm{C}_{\mathrm{G}}(\Gamma)$. Let $\mathcal{S}=\Gamma+\mathfrak{g}_{x, r^{+}}$ and $\mathcal{S}^{\prime}=\Gamma^{\prime}+\mathfrak{g}_{x^{\prime}, r^{+}}$be two good cosets. If $\mathcal{S} \sim \mathcal{S}^{\prime}$, then there exist $g \in G$ and $y \in \mathcal{B}\left(\mathrm{G}^{\prime}, k\right)$ such that ${ }^{g}\left(\Gamma^{\prime}+\mathfrak{g}_{x^{\prime}, r^{+}}\right) \subset \Gamma+\mathfrak{g}_{y, r^{+}}$.

Proof. - By Lemma 1.3.4, there exist $h \in G_{x, 0^{+}}$and $n^{\prime} \in \mathfrak{g}_{h x^{\prime}, r}^{\prime} \cap \mathcal{N}$ such that $h x^{\prime} \in \mathcal{B}\left(\mathrm{G}^{\prime}, k\right)$ and ${ }^{h}\left(\Gamma^{\prime}+\mathfrak{g}_{x^{\prime}, r^{+}}\right)=\Gamma+n^{\prime}+\mathfrak{g}_{h x^{\prime}, r^{+}}$. Now, by Lemma 1.3.2, there 
exist $h^{\prime} \in G_{h x^{\prime}, 0}^{\prime}$ and $y \in \mathcal{B}\left(G^{\prime}, k\right)$ such that ${ }^{h^{\prime} h}\left(\Gamma^{\prime}+\mathfrak{g}_{x^{\prime}, r^{+}}\right)=\Gamma+{ }^{h^{\prime}} n^{\prime}+\mathfrak{g}_{h x^{\prime}, r^{+}} \subset$ $\Gamma+\mathfrak{g}_{y, r^{+}}$.

As usual, we use the same notation for an equivalence class and its representative when this will not lead to any confusion.

Definition 1.4.3. - For $\mathcal{S} \in \mathfrak{S}$, define $\mathfrak{g}_{\mathcal{S}} \subset \mathfrak{g}$ as follows:

$$
\mathfrak{g}_{\mathcal{S}}:=\bigcup_{\mathcal{S}^{\prime} \sim \mathcal{S}} G_{\mathcal{S}^{\prime}} .
$$

EXAMPLE 1.4.4. - If $\mathcal{S}$ is a 0-good set, then $\mathfrak{g}_{\mathcal{S}}=\mathfrak{g}_{0}$. If $\mathcal{S}=\Gamma+\mathfrak{g}_{x, r^{+}}$, from Lemma 1.4.2 and Remark 1.3.3, 1), we have $\mathfrak{g}_{\mathcal{S}}=G\left(\Gamma+\mathfrak{g}_{r^{+}}^{\prime}\right)$. The second example is due to F. Murnaghan.

The following lemma shows that the weak associativity on good cosets induces a partition of $\mathfrak{g}$, and in turn, a spectral decomposition of the delta distribution on $\mathfrak{g}$.

Lemma 1.4.5. - Suppose (HGT) holds. Then,

1) $\mathfrak{g}$ is the disjoint union of $\mathfrak{g}_{\mathcal{S}}, \mathcal{S} \in \mathfrak{S}$ :

$$
\mathfrak{g}=\bigcup_{\mathcal{S} \in \mathfrak{S}}^{\circ} \mathfrak{g}_{\mathcal{S}} .
$$

2) Each $\mathfrak{g}_{\mathcal{S}}$ is a $G$-domain, that is, a $G$-invariant open and closed subset of $\mathfrak{g}$ in the $p$-adic topology.

3) For any $f \in C_{c}^{\infty}(\mathfrak{g})$, we can decompose $\int_{\mathfrak{g}} f(X) \mathrm{d} X$ as follows:

$$
\int_{\mathfrak{g}} f(X) \mathrm{d} X=\sum_{\mathcal{S} \in \mathfrak{S}} \int_{\mathfrak{g}_{\mathcal{S}}} f(X) \mathrm{d} X .
$$

Proof. - Part 3) is a consequence of 1) and 2). To prove 1), let $X \in \mathfrak{g}$ with Jordan decomposition $X_{s}+X_{n}$. Let T be a maximal $k$-torus containing $X_{s}$ with $\mathrm{d}\left(X_{s}\right)=r$. If $r \geq 0, X \in \mathfrak{g}_{\mathcal{S}}$ where $\mathcal{S}$ is a 0 -good coset. If $r<0$, from (HGT), there is a good element $\Gamma$ in $\mathfrak{t}_{r} \backslash \mathfrak{t}_{r}+$ such that $\Gamma \equiv X_{s}\left(\bmod \mathfrak{g}_{x, r^{+}}\right)$ for any $x \in \mathcal{B}\left(\mathrm{C}_{\mathrm{G}}\left(X_{s}\right), k\right)$. Since $X_{n} \in C_{G}\left(X_{s}\right)$ and $\mathrm{d}\left(X_{n}\right)=\infty$, one can find $y \in \mathcal{B}\left(\mathrm{C}_{\mathrm{G}}\left(X_{s}\right), k\right)$ such that $X_{n} \in \mathfrak{g}_{y, r^{+}}$. Then since $y \in \mathcal{B}\left(\mathrm{C}_{\mathrm{G}}\left(X_{s}\right), k\right) \subset$ $\mathcal{B}\left(\mathrm{C}_{\mathrm{G}}(\Gamma), k\right)$, we see $X=X_{s}+X_{n}$ is contained in a good coset $\Gamma+\mathfrak{g}_{y, r^{+}}$.

To prove 2), observe that $\mathfrak{g}_{\mathcal{S}}$ is obviously $G$-invariant. Each $\mathfrak{g}_{\mathcal{S}}$ is open since each $\mathcal{S}$ is open and $\mathfrak{g}_{\mathcal{S}}$ is the union of ${ }^{G} \mathcal{S}^{\prime}$ with $\mathcal{S}^{\prime} \sim \mathcal{S}$. It is closed because its complement is a union of open sets from 1 ).

BULLETIN DE LA SOCIÉtÉ MATHÉMATIQUE DE FRANCE 
REMARK 1.4.6. - From Remark 1.2.3, if $\mathcal{S} \in \mathfrak{S}$ is not a 0 -good coset, we have for any $X \in \mathfrak{g}_{\mathcal{S}}, \mathrm{d}(X)=\mathrm{d}(\mathcal{S})$. If hypothesis (HGT) holds, we also observe that

$$
\mathfrak{g}_{\mathcal{S}} \subset\left(\mathfrak{g}_{\mathrm{d}(\mathcal{S})} \backslash \mathfrak{g}_{\mathrm{d}(\mathcal{S})^{+}}\right)=\bigcup_{\substack{\mathcal{S}^{\prime} \in \mathfrak{S} \\ \mathrm{d}\left(\mathcal{S}^{\prime}\right)=\mathrm{d}(\mathcal{S})}} \mathfrak{g}_{\mathcal{S}^{\prime}} .
$$

\section{Unrefined minimal K-types and $\widetilde{G}$}

2.1. Unrefined minimal K-types and dual blobs. - Here, we recall some results from [14], [15], and define dual blobs. For the purpose of simpler explanation, as in [14], we assume that there is a natural isomorphism

$$
\phi: G_{x, r} / G_{x, r^{+}} \longrightarrow \mathfrak{g}_{x, r} / \mathfrak{g}_{x, r^{+}}
$$

when $r>0$. By [20, 2.4], such an isomorphism exists whenever G splits over a tamely ramified extension of $k$ (see also $[1,1.6]$ ).

DEFINITION 2.1.1 (see [14], [15]). — An unrefined minimal K-type (or minimal K-type) is a pair $\left(G_{x, \varrho}, \chi\right)$, where $x \in \mathcal{B}(\mathrm{G}, k)$, $\varrho$ is a nonnegative real number, $\chi$ is a representation of $G_{x, \varrho}$ trivial on $G_{x, \varrho^{+}}$and

(i) if $\varrho=0, \chi$ is a cuspidal representation of $G_{x, 0} / G_{x, 0^{+}}$inflated to $G_{x, 0}$;

(ii) if $\varrho>0, \chi$ is a nondegenerate character of $G_{x, \varrho} / G_{x, \varrho^{+}}$.

The $\varrho$ in the above definition is called the depth of the minimal K-type $\left(G_{x, \varrho}, \chi\right)$.

Definition 2.1.2 (see $[13,2.3 .4])$. — Let $\mathfrak{s}=\left(G_{x, \varrho}, \chi\right)$ be a minimal K-type. If $\varrho>0$, we call the coset $\mathcal{S}=\Gamma^{*}+\mathfrak{g}_{x,(-\varrho)^{+}}$a dual blob of $\mathfrak{s}$ when $\chi$ is realized by $\mathcal{S}$. That is, for any $g \in G_{x, \varrho}$,

$$
\chi(g)=\Lambda\left(\Gamma^{*}(\phi(g))\right) .
$$

If $\varrho=0$, we define the dual blob of $\mathfrak{s}$ to be $\mathfrak{g}_{x, 0}^{*}$. We denote by $\mathfrak{s}^{\sharp}$ the dual blob of $\mathfrak{s}$.

If $\varrho>0$, any coset $X^{*}+\mathfrak{g}_{x,(-\varrho)^{+}}^{*}$ in $\mathfrak{g}_{x,-\varrho}^{*}$ defines a character of $G_{x, \varrho}$. We denote by $\chi_{X^{*}}$ the character of $G_{x, \varrho}$ represented by $X^{*}+\mathfrak{g}_{x,(-\varrho)}^{*}+$ when there is no confusion.

Recall that a coset $X^{*}+\mathfrak{g}_{x,(-\varrho)^{+}}^{*}$ in $\mathfrak{g}^{*}$ is nondegenerate if $X^{*}+\mathfrak{g}_{x,(-\varrho)^{+}}^{*}$ does not contain any nilpotent element. A character $\chi$ of $G_{x, \varrho}$ is nondegenerate if the dual blob of $\left(G_{x, \varrho}, \chi\right)$ is nondegenerate.

Definition 2.1.3 (see [14]). - Two minimal K-types $\mathfrak{s}=\left(G_{x, r}, \chi\right)$ and $\mathfrak{s}^{\prime}=$ $\left(G_{y, s}, \xi\right)$ are said to be associates if they have the same depth, and

1) if $r=0$, there is a $g \in G$ such that $G_{x, 0} \cap G_{g y, 0}$ surjects onto both $G_{x, 0} / G_{x, 0^{+}}$and $G_{g y, 0} / G_{g y, 0^{+}}$, and $\chi$ is isomorphic to $\xi^{g}$;

2) if $r>0$, the $G$-orbit of the dual blob $\mathfrak{s}^{\sharp}$ intersects with $\mathfrak{s}^{\sharp}$.

TOME $132-2004-\mathrm{N}^{\mathrm{O}} 1$ 
TheOREM 2.1 .4 (see $[15,3.5])$. - Given $\left(\pi, V_{\pi}\right) \in \widetilde{G}$, there is a nonnegative rational number $\varrho(\pi)$ with the following properties.

1) For some $x \in \mathcal{B}(\mathrm{G}, k)$, the space $V_{\pi}^{G_{x, \varrho(\pi)+}}$ of $G_{x, \varrho(\pi)^{+-}}$fixed vectors is nonzero and $\varrho(\pi)$ is the smallest number with this property.

2) For any $y \in \mathcal{B}(\mathrm{G}, k)$, if $W=V_{\pi}^{G_{y, \varrho(\pi)}+} \neq\{0\}$, then

(i) if $\varrho(\pi)=0$, any irreducible $G_{y, \varrho(\pi)}$-submodule of $W$ contains a minimal K-type of depth zero of a parahoric $G_{z, 0} \subset G_{y, 0}$;

(ii) if $\varrho(\pi)>0$, any irreducible $G_{y, \varrho(\pi)}$-submodule of $W$ is a minimal $\mathrm{K}$-type.

Moreover, any two minimal $\mathrm{K}$-types contained in $\pi$ are associates of each other.

The rational number $\varrho(\pi)$ in the theorem is called the depth of the irreducible admissible representation $\left(\pi, V_{\pi}\right)$.

\subsection{Partition of $\widetilde{G}$ via minimal K-types}

Definition 2.2.1. - Assume the hypothesis (HB) holds. Let $\mathfrak{s}$ and $\mathfrak{s}^{\prime}$ be two minimal K-types of depth $\varrho$. We say $\mathfrak{s}$ is weakly associated to $\mathfrak{s}^{\prime}$ if there is a sequence of minimal K-types of $\mathfrak{s}=\mathfrak{s}_{0}, \mathfrak{s}_{1}, \ldots, \mathfrak{s}_{k}=\mathfrak{s}^{\prime}$ depth $\varrho$ such that the dual blobs $\mathfrak{s}_{i}^{\sharp}$ and $\mathfrak{s}_{i+1}^{\sharp}$ of $\mathfrak{s}_{i}$ and $\mathfrak{s}_{i+1}$ satisfy that $\mathfrak{s}_{i}^{\sharp} \cap{ }^{g_{i}} \mathfrak{s}_{i+1}^{\sharp} \neq \varnothing$ for some $g_{i} \in G$.

Recall we defined the dual blob of a minimal K-type $\left(G_{x, 0}, \sigma\right)$ to be $\mathfrak{g}_{x, 0}$. Hence any two types of depth zero are weakly associated, but they are not necessarily associated. It is straightforward that the above weak associativity is an equivalence relation. On the other hand, associativity is not an equivalence relation.

We denote by $\mathfrak{S}_{K}$ the set of equivalence classes of weakly associated minimal K-types.

REMARK 2.2.2. - Let $\mathfrak{s}$ be a minimal K-type, and let $\overline{\mathfrak{s}} \in \mathfrak{S}_{\mathrm{K}}$ be its equivalence class. Let $\widetilde{G}_{\mathfrak{s}}$ be the set of $\left(\pi, V_{\pi}\right) \in \widetilde{G}$ which contain a minimal K-type weakly associated to $\mathfrak{s}$. Note that for any $\mathfrak{s}^{\prime} \in \overline{\mathfrak{s}}$, we have $\widetilde{G}_{\mathfrak{s}}=\widetilde{G}_{\mathfrak{s}^{\prime}}$. Then from Theorem 2.1.4, we have

$$
\widetilde{G}=\bigcup_{\mathfrak{s} \in \mathfrak{S}_{\mathrm{K}}}^{\circ} \widetilde{G}_{\mathfrak{s}} .
$$

Note that if $\mathfrak{s}$ is a minimal K-type of depth zero, $\widetilde{G}_{\mathfrak{s}}$ is the set of all depth zero irreducible representations.

From now on, we use the same notation for an equivalence class and its representative in cases where there is no confusion.

BULlETIN DE LA SOCIÉTÉ MATHÉMATIQUE DE FRANCE 


\section{Plancherel distributions and good minimal K-types}

In this section, we investigate some relations between partitions of $\mathfrak{g}$ and $\widetilde{G}$. These partitions are discussed in the previous sections. As stated in Theorem 3.3.1, they give rise to a matching between spectral decomposition factors. We first review Plancherel formulas.

3.1. Plancherel formulas on $\mathfrak{g}$ and $\boldsymbol{G}$. - For $\left(\pi, V_{\pi}\right) \in \widehat{G}$ and $f \in C_{c}^{\infty}(G)$, we define a function on $\widehat{G}$ as

$$
\widehat{f}(\pi):=\operatorname{Tr}(\pi(f))=\Theta_{\pi}(f) .
$$

Then Harish-Chandra's Plancherel formula (see [7]) states that there is a Borel measure $\mathrm{d} \pi$ supported on $\widehat{G}$ such that

$$
f(1)=\int_{\widehat{G}} \widehat{f}(\pi) \mathrm{d} \pi .
$$

On the other hand, regarding $\mathfrak{g}$ as a topological group with respect to addition, we have the following isomorphisms:

$$
\widehat{\mathfrak{g}} \simeq \mathfrak{g}^{*} \simeq \mathfrak{g} .
$$

The first isomorphism is from Pontrjagin duality, and we also have the second isomorphism via the additive character $\Lambda$ and an appropriate bilinear form on $\mathfrak{g}$. Then the Plancherel formula on $\mathfrak{g}$ can be formulated as follows: there is an appropriate measure on $\widehat{\mathfrak{g}}$ such that for $f \in C_{c}^{\infty}(\mathfrak{g})$,

$$
f(0)=\int_{\widehat{\mathfrak{g}}} \widehat{f}(\chi) \mathrm{d} \chi,
$$

where $\widehat{f} \in C_{c}^{\infty}(\widehat{\mathfrak{g}})$ is the Fourier transformation of $f$ given by $\widehat{f}(\chi)=$ $\int_{\widehat{\mathfrak{g}}} f(Y) \chi(Y) \mathrm{d} Y$. When $(\mathrm{HB})$ holds, from the identification $\widehat{\mathfrak{g}} \simeq \mathfrak{g}$, we can now write

$$
f(0)=\int_{\mathfrak{g}} \widehat{f}(X) \mathrm{d} X
$$

where $\widehat{f}(X)=\int_{\mathfrak{g}} f(Y) \Lambda(\mathrm{B}(X, Y)) \mathrm{d} Y$. In (4), the $G$-invariant measure $\mathrm{d} X$ on $\mathfrak{g}$ should satisfy $\operatorname{vol}_{\mathfrak{g}}\left(\mathfrak{g}_{x, r}\right) \operatorname{vol}_{\mathfrak{g}}\left(\mathfrak{g}_{x,(-r)^{+}}\right)=1$ for all $x \in \mathcal{B}(\mathrm{G}, k)$ and $r \in \mathbb{R}$.

To relate Plancherel formulas on $\mathfrak{g}$ and $G$, let $f \in C_{c}^{\infty}(\mathfrak{g})$ be supported in a sufficiently small neighborhood of 0 . Then $f \circ \log$ defines a function in $C_{c}^{\infty}(G)$. Combining the Plancherel formulas (3) and (4), we have

$$
\int_{\mathfrak{g}} \widehat{f}(X) \mathrm{d} X=f(0)=\int_{\widehat{G}} \Theta_{\pi}(f \circ \log ) \mathrm{d} \pi .
$$

TOME $132-2004-\mathrm{N}^{\mathrm{O}} 1$ 


\subsection{Good cosets, good minimal K-types and $\widetilde{G}$}

Definition 3.2.1 (see [3], [13]). - Assume (HB) is valid. Let $\mathfrak{s}=\left(G_{x, \varrho}, \chi\right)$ be a minimal K-type. We say $\left(G_{x, \varrho}, \chi\right)$ is good, if its dual blob $\mathfrak{s}^{\sharp}$ is a good coset.

Note that all minimal K-types of depth zero are good. For later use, we record the following corollary of Lemma 1.3.2:

Corollary 3.2.2. - We keep the notation from Lemma 1.3.2. Assume (HB) is valid. Suppose $\varrho:=-r>0$. If $\left(\pi, V_{\pi}\right) \in \widetilde{G}$ contains a minimal $\mathrm{K}$-type $\left(G_{x, \varrho}, \chi_{\Gamma+X^{\prime}}\right)$ with dual blob $\Gamma+X^{\prime}+\mathfrak{g}_{x, r^{+}}$, it also contains a good minimal K-type $\left(G_{y, \varrho}, \chi_{\Gamma}\right)$ with dual blob $\Gamma+\mathfrak{g}_{y, r^{+}}$.

Definition 3.2.3. - Assume (HB) holds. Let $\mathcal{S} \in \mathfrak{S}$ and let $\mathfrak{s}=\left(G_{x, \varrho}, \chi\right)$ be a minimal K-type.

1) If $\mathfrak{s}^{\sharp} \sim \mathcal{S}$, we say that $\mathfrak{s}$ is weakly associated to $\mathcal{S}$.

2) Define the subset $\widetilde{G}_{\mathcal{S}}$ of $\widetilde{G}$ to be the set of all $\left(\pi, V_{\pi}\right) \in \widetilde{G}$ such that $\left(\pi, V_{\pi}\right)$ contains a good minimal K-type $\mathfrak{s}$ with $\mathfrak{s}^{\sharp} \sim \mathcal{S}$. We also define

$$
\widehat{G}_{\mathcal{S}}:=\widetilde{G}_{\mathcal{S}} \cap \widehat{G} .
$$

Remarks 3.2.4. - 1) If $\mathcal{S}$ is a 0 -good coset, $\widetilde{G}_{\mathcal{S}}$ is the set of depth zero representations. Otherwise, the good minimal K-types contained in the representations in $\widetilde{G}_{\mathcal{S}}$ are weakly associated to each other. In particular, the depth of any representation in $\widetilde{G}_{\mathcal{S}}$ is $-\mathrm{d}(\mathcal{S})$.

2) If $\mathfrak{s}$ is a good K-type weakly associated to $\mathcal{S}$, we have $\widetilde{G}_{\mathcal{S}} \subset \widetilde{G}_{\mathfrak{s}}$. This is a direct consequence of the Definition 2.2.1. We observe

$$
\widetilde{G}_{\mathcal{S}}=\left\{\left(\pi, V_{\pi}\right) \in \widetilde{G}_{\mathfrak{s}} \mid\left(\pi, V_{\pi}\right) \text { contains a good minimal K-type }\right\} .
$$

3) Observe that if $\mathcal{S} \sim \mathcal{S}^{\prime}$, then $\widetilde{G}_{\mathcal{S}}=\widetilde{G}_{\mathcal{S}^{\prime}}$. Hence, for $\mathcal{S} \in \mathfrak{S}$, we can write $\widetilde{G}_{\mathcal{S}}$ without any confusion.

REMARK 3.2.5. - Although we will later give another proof, we recall from Theorem 2.4.10 of [13] that any $\left(\pi, V_{\pi}\right) \in \widetilde{G}$ contains a good K-type. Combining this with Theorem 2.1.4 implies the following: Suppose (HB) and (HGT) are valid. Let $\mathcal{S}_{1}, \mathcal{S}_{2} \in \mathfrak{S}$ and $\left(\pi, V_{\pi}\right) \in \widetilde{G}$.

(i) If $\left(\pi, V_{\pi}\right)$ contains minimal $\mathrm{K}$-types $\mathfrak{s}_{1}, \mathfrak{s}_{2}$ with $\mathfrak{s}_{i} \sim \mathcal{S}_{i}, i=1,2$, then we have $\mathcal{S}_{1} \sim \mathcal{S}_{2}$.

(ii) The $\widetilde{G}$ is a disjoint union of $\widetilde{G}_{\mathcal{S}}$ 's with $\mathcal{S} \in \mathfrak{S}: \widetilde{G}=\bigcup_{\mathcal{S} \in \mathfrak{S}} \widetilde{G}_{\mathcal{S}}$.

Lemma 3.2.6. - Suppose (HB) is valid.

1) For $\mathcal{S} \in \mathfrak{S}, \widehat{G}_{\mathcal{S}}$ is open and closed in $\widehat{G}$. 
2) For $f \in C_{c}^{\infty}(G)$, we have

$$
\int_{\widehat{G}} \widehat{f}(\pi) \mathrm{d} \pi=\int_{\widehat{G} \backslash\left(\bigcup_{\mathcal{S} \in \mathfrak{S}} \widehat{G}_{\mathcal{S}}\right)} \widehat{f}(\pi) \mathrm{d} \pi+\sum_{\mathcal{S} \in \mathfrak{S}} \int_{\widehat{G}_{\mathcal{S}}} \widehat{f}(\pi) \mathrm{d} \pi .
$$

3) Each $\widehat{G}_{\mathcal{S}}$ has a finite Plancherel volume, that is, $\operatorname{vol}_{\widehat{G}}\left(\widehat{G}_{\mathcal{S}}\right)<\infty$.

Proof. - 1) Assume $\mathrm{d}(\mathcal{S})<0$ first. We fix a good element $\Gamma$ such that $\mathcal{S}=$ $\Gamma+\mathfrak{g}_{x,(-\varrho)^{+}}$with $x \in \mathcal{B}\left(\mathrm{C}_{\mathrm{G}}(\Gamma), k\right)$. For $\mathcal{S}^{\prime} \sim \mathcal{S}$, let $\mathfrak{s}_{\mathcal{S}^{\prime}}=\left(J_{\mathcal{S}^{\prime}}, \chi_{\mathcal{S}^{\prime}}\right)$ be the good minimal K-type with dual blob $\mathcal{S}^{\prime}$. Let $\Delta$ be the set of $G$-conjugacy classes of $\mathfrak{s}_{\mathcal{S}^{\prime}}$ with $\mathcal{S}^{\prime} \sim \mathcal{S}$. Let

$$
\widehat{G}\left(\mathcal{S}^{\prime}\right):=\left\{\left(\pi, V_{\pi}\right) \in \widehat{G} \mid m\left(\chi_{\mathcal{S}^{\prime}}, V_{\pi}\right)>0\right\},
$$

where $m\left(\chi_{\mathcal{S}^{\prime}}, V_{\pi}\right)$ is the multiplicity of $\chi_{\mathcal{S}^{\prime}}$ in $\left(\pi, V_{\pi}\right) \in \widehat{G}$. Note that (i) each $\widehat{G}\left(\mathcal{S}^{\prime}\right)$ is open and closed because $m\left(\chi_{\mathcal{S}^{\prime}}, V_{\pi}\right)$ is semicontinuous on $\widehat{G}$, (ii) if $\mathcal{S}^{\prime}$ and $\mathcal{S}^{\prime \prime}$ are $G$-conjugates, $\widehat{G}\left(\mathcal{S}^{\prime}\right)=\widehat{G}\left(\mathcal{S}^{\prime \prime}\right)$, (iii) $\Delta$ is finite, and (iv) $\widehat{G}_{\mathcal{S}}=\bigcup_{\mathcal{S}^{\prime} \in \Delta} \widehat{G}\left(\mathcal{S}^{\prime}\right)$. Hence $\widehat{G}_{\mathcal{S}}$ is open and closed when $\mathrm{d}(\mathcal{S})<0$. If $\mathcal{S}$ is a 0 -good coset, we have

$$
\widehat{G}_{\mathcal{S}}=\left\{\left(\pi, V_{\pi}\right) \in \widehat{G} \mid V_{\pi}^{G_{x, 0^{+}}} \neq 0, \text { for some } x \in \mathcal{B}(\mathrm{G}, k)\right\} .
$$

Then this case can be proved in a similar way.

2) This is a consequence of 1 ).

3) More generally, it is enough to show that for any open compact subgroup $J \subset G$, the set $\widehat{G}^{J}$ defined by $\left\{\left(\pi, V_{\pi}\right) \in \widehat{G} \mid V_{\pi}^{J} \neq 0\right\}$ has a finite Plancherel volume. It follows from the following sequence of inequalities: for $f \in C_{c}^{\infty}(G)$ given by the characteristic function of $J$ divided by $\operatorname{vol}_{G}(J)$, from the Plancherel formula on $G$, we have

$$
\begin{aligned}
\operatorname{vol}_{\widehat{G}}\left(\widehat{G}^{J}\right) \leq \int_{\widehat{G}^{J}} \operatorname{dim}_{\mathbb{C}}\left(V_{\pi}^{J}\right) \mathrm{d} \pi & =\int_{\widehat{G}} \operatorname{dim}_{\mathbb{C}}\left(V_{\pi}^{J}\right) \mathrm{d} \pi=\int_{\widehat{G}} \widehat{f}(\pi) \mathrm{d} \pi \\
& =f(1)=\frac{1}{\operatorname{vol}_{G}(J)} .
\end{aligned}
$$

3.3. Main Theorem. - The following is a refinement of the equality $(\mathrm{Pl})$. That is, we find an equality between summands of the left and right hand sides (see Lemma 1.4.5 and Lemma 3.2.6) of the equation. The proof will be given in the following section.

THEOREM 3.3.1. - Suppose hypothesis $(\mathrm{H} k)$ is valid. Let $\mathcal{S}$ be a good coset and $f \in C_{c}^{\infty}(\mathfrak{g})$. If $\mathcal{S}$ is a 0 -good coset, assume $f \in C_{c}^{\infty}\left(\mathfrak{g}_{0^{+}}\right)$. If $\mathcal{S}$ is a good coset of depth $-\varrho<0$, assume $f \in C_{c}^{\infty}\left(\mathfrak{g}_{\varrho}\right)$. Then, we have

$$
\int_{\mathfrak{g}_{\mathcal{S}}} \widehat{f}(X) \mathrm{d} X=\int_{\widehat{G}_{\mathcal{S}}} \widehat{f \circ \log }(\pi) \mathrm{d} \pi .
$$

TOME $132-2004-\mathrm{N}^{\mathrm{O}} 1$ 


\section{Proof and applications of Theorem 3.3.1}

In this section, we prove Theorem 3.3.1. We treat positive depth cases and depth zero cases separately. Before each case, we recall some relevant results from [13] and from [4], [5] respectively.

Recall a distribution $\mathrm{T}$ on $C_{c}^{\infty}(\mathfrak{g})$ is a $\mathbb{C}$-valued linear functional on $C_{c}^{\infty}(\mathfrak{g})$. The distribution $\mathrm{T}$ is called invariant if ${ }^{g} \mathrm{~T}=\mathrm{T}$ for $g \in G$, where ${ }^{g} f(X)=$ $f\left(g^{-1} X\right)$ and ${ }^{g} \mathrm{~T}(f)=\mathrm{T}\left({ }^{g^{-1}} f\right)$ for $f \in C_{c}^{\infty}(\mathfrak{g})$. We denote the space of invariant distributions by $\mathcal{J}(\mathfrak{g})$. For any subset $\mathcal{L} \subset \mathfrak{g}$, we denote the subspace of invariant distributions supported on the closure of ${ }^{G} \mathcal{L}$ by $\mathcal{J}(\mathcal{L})$. For $r \in \mathbb{R}$, we define some $G$-invariant subspaces of $C_{c}^{\infty}(\mathfrak{g})$ as follows:

$$
\mathcal{D}_{r}:=\sum_{x \in \mathcal{B}(\mathrm{G}, k)} C_{c}\left(\mathfrak{g} / \mathfrak{g}_{x, r}\right), \quad \mathcal{D}_{r^{+}}:=\sum_{x \in \mathcal{B}(\mathrm{G}, k)} C_{c}\left(\mathfrak{g} / \mathfrak{g}_{x, r^{+}}\right)
$$

For any $\mathrm{T} \in \mathcal{J}(\mathfrak{g})$ and $\mathcal{D} \subset C_{c}^{\infty}(\mathfrak{g})$, we denote the restriction of $\mathrm{T}$ to $\mathcal{D}$ by $\operatorname{res}_{\mathcal{D}} \mathrm{T}$. For any $\mathcal{J} \subset \mathcal{J}(\mathfrak{g})$, let $\operatorname{res}_{\mathcal{D}} \mathcal{J}$ denote $\left\{\operatorname{res}_{\mathcal{D}} \mathrm{T} \mid \mathrm{T} \in \mathcal{J}\right\}$.

\subsection{Some results on character expansions: positive depth cases}

Recall from $[13,3.1 .2]$ that we defined some subspaces of $\mathcal{J}(\mathfrak{g})$ to characterize the character distributions of those $\pi$ which contain a given good minimal Ktype.

Definition 4.1.1 (see [13, 3.1.2]). - Let $\Gamma$ be a good element of depth $r<0$ (note that $\Gamma \neq 0$ ). Let $\mathrm{G}^{\prime}:=\mathrm{C}_{\mathrm{G}}(\Gamma)$ be the centralizer of $\Gamma$ in $\mathrm{G}$, and let $\mathcal{N}^{\prime}:=\mathcal{N} \cap \mathfrak{g}^{\prime}$, the set of nilpotent elements in $\mathfrak{g}^{\prime}$. We define some subspaces of $\mathcal{J}(\mathfrak{g})$ :

1) For $x \in \mathcal{B}(\mathrm{G}, k)$ and $r \in \mathbb{R}$, we define $\mathcal{J}_{x, s, r^{+}}^{\Gamma}$ as follows:

$$
\begin{aligned}
& \text { - If } s<r, \quad \mathcal{J}_{x, s, r^{+}}^{\Gamma}:=\left\{\begin{array}{l|l}
\mathrm{T} \in \mathcal{J}(\mathfrak{g}) & \begin{array}{l}
\text { for } f \in C\left(\mathfrak{g}_{x, s} / \mathfrak{g}_{x, r^{+}}\right) \\
\text {if } \operatorname{Supp}(f) \cap\left(\mathcal{N}+\mathfrak{g}_{x, s^{+}}\right)=\varnothing \\
\text { then } \mathrm{T}(f)=0
\end{array}
\end{array}\right\} . \\
& \text { - If } s=r, \quad \mathcal{J}_{x, r, r^{+}}^{\Gamma}:=\left\{\begin{array}{l|l}
\mathrm{T} \in \mathcal{J}(\mathfrak{g}) & \begin{array}{l}
\text { for } f \in C\left(\mathfrak{g}_{x, r} / \mathfrak{g}_{x, r^{+}}\right) \\
\text {if } \operatorname{Supp}(f) \cap{ }^{G}\left(\Gamma+\mathcal{N}^{\prime}\right)=\varnothing \\
\text { then } \mathrm{T}(f)=0
\end{array}
\end{array}\right\} \text {. }
\end{aligned}
$$

2) $\quad \mathcal{J}_{r^{+}}^{\Gamma}:=\bigcap_{x \in \mathcal{B}(\mathrm{G}, k)} \bigcap_{s \leq r} \mathcal{J}_{x, s, r^{+}}^{\Gamma}$

For $X \in \mathfrak{g}$, let $\mathcal{O}(X)$ be the set of all $G$-orbits whose closures contain $X$. For $X \in \mathfrak{g}^{\prime}, \mathcal{O}^{\prime}(X)$ denotes the set of all $G^{\prime}$-orbits whose closures in $\mathfrak{g}^{\prime}$ contain $X$. Recall that there is a bijection between $\mathcal{O}^{\prime}(0)$ and $\mathcal{O}(\Gamma)$ given by $n^{\prime} \leftrightarrow \Gamma+n^{\prime}$ (see [8]).

For each $\mathcal{O} \in \mathcal{O}(\Gamma)$, let $\mu_{\mathcal{O}}$ be the orbital integral associated to $\mathcal{O}$. Denote the span of $\mu_{\mathcal{O}}$ with $\mathcal{O} \in \mathcal{O}(\Gamma)$ by $\mathcal{J}_{\Gamma}$.

BULletin DE LA SOCiÉtÉ MATHÉMATiQUE DE FRANCE 
Proposition 4.1.2. - Suppose $(\mathrm{H} k)$ holds. Let $\Gamma$ be a good element of depth $r<0$, and let $\mathrm{G}^{\prime}:=\mathrm{C}_{\mathrm{G}}(\Gamma)$. Then we have

1) $\operatorname{res}_{\mathcal{D}_{r}+} \mathcal{J}_{r^{+}}^{\Gamma}=\operatorname{res}_{\mathcal{D}_{r}+} \mathcal{J}_{\Gamma}$

2) for $\mathrm{T}_{1}, \mathrm{~T}_{2} \in \mathcal{J}_{r^{+}}^{\Gamma}, \operatorname{res}_{\mathcal{D}_{r}+} \mathrm{T}_{1}=\operatorname{res}_{\mathcal{D}_{r}+} \mathrm{T}_{2}$ if and only if

$$
\mathrm{T}_{1}\left(\left[\Gamma+v+\mathfrak{g}_{x, r^{+}}\right]\right)=\mathrm{T}_{2}\left(\left[\Gamma+v+\mathfrak{g}_{x, r^{+}}\right]\right)
$$

for each $x \in \mathcal{B}\left(\mathrm{G}^{\prime}, k\right)$ and $v \in \mathcal{N}^{\prime} \cap \mathfrak{g}_{x, r}$.

Recall that $\left[\Gamma+v+\mathfrak{g}_{x, r^{+}}\right]$is the characteristic function supported on the set $\Gamma+v+\mathfrak{g}_{x, r^{+}}$.

Proof. - Part 1) follows from [13, 3.1.7]. Part 2) is a corollary of 3.1 .7 and 4.2.1 in [13].

In fact, 2) is a weaker statement of the results in [13]. However, 2) is enough for our purposes, and we can avoid introducing new notation included in the stronger statement.

TheOrEm 4.1 .3 (see $[13,5.2 .1,5.3 .1])$. - Assume that $(\mathrm{H} k)$ is valid. Suppose that $\left(\pi, V_{\pi}\right)$ is an irreducible admissible representation of $G$ of positive depth $\varrho(\pi)=\varrho$. Suppose $\left(\pi, V_{\pi}\right)$ contains a good minimal $\mathrm{K}$-type $\mathfrak{s}=\left(G_{x_{0}, \varrho}, \chi_{\Gamma}\right)$. Let $\Gamma+\mathfrak{g}_{x_{0},(-\varrho)^{+}}$be the dual blob of $\mathfrak{s}$ containing a good element $\Gamma$. Then,

1) $\widehat{\Theta}_{\pi} \in \mathcal{J}_{(-\varrho)^{+}}^{(-\Gamma)}$,

2) $\Theta_{\pi}$ is $\Gamma$-asymptotic on $\mathfrak{g}_{\varrho}$. That is, there are $c_{\mathcal{O}}(\pi) \in \mathbb{C}$ indexed by $\mathcal{O}(\Gamma)$, such that for any regular element $X \in \mathfrak{g}_{\varrho}$,

$$
\Theta_{\pi}(\exp (X))=\sum_{\mathcal{O} \in \mathcal{O}(\Gamma)} c_{\mathcal{O}}(\pi) \cdot \widehat{\mu_{\mathcal{O}}}(X)
$$

4.2. Proof of Theorem 3.3.1: positive depth cases. - Fix $\varrho>0$. We keep the notation from the previous section. Let $\mathrm{T}_{\mathcal{S}}^{1}$ and $\mathrm{T}_{\mathcal{S}}^{2}$ be invariant distributions defined as follows: for $f \in C_{c}^{\infty}(\mathfrak{g})$, denote by $f_{0^{+}}$the function $f \cdot\left[\mathfrak{g}_{0^{+}}\right]$where $\left[\mathfrak{g}_{0^{+}}\right]$is the characteristic function supported on $\mathfrak{g}_{0^{+}}$. Then

$$
\begin{aligned}
& \mathrm{T}_{\mathcal{S}}^{1}(f):=\int_{\mathfrak{g}_{\mathcal{S}}} \widehat{f_{0^{+}}}(X) \mathrm{d} X \\
& \mathrm{~T}_{\mathcal{S}}^{2}(f):=\int_{\widehat{G}_{\mathcal{S}}} \widehat{f_{0^{+}} \circ \log }(\pi) \mathrm{d} \pi=\int_{\widehat{G}_{\mathcal{S}}} \Theta_{\pi}\left(f_{0^{+}} \circ \log \right) \mathrm{d} \pi .
\end{aligned}
$$

Then to prove the theorem, it is enough to prove that

$$
\mathrm{T}_{\mathcal{S}}^{1}(f)=\mathrm{T}_{\mathcal{S}}^{2}(f)
$$

for any $f \in C_{c}^{\infty}\left(\mathfrak{g}_{\varrho}\right)$ when $\mathrm{d}(\mathcal{S})=-\varrho$.

TOME $132-2004-\mathrm{N}^{\mathrm{O}} 1$ 
On the other hand, the Fourier transform maps $C_{c}^{\infty}\left(\mathfrak{g}_{\varrho}\right)$ to $\mathcal{D}_{(-\varrho)}+($ see $[2$, 4.2.3]). We will prove Theorem 3.3 .1 by showing that $\widehat{T}_{\mathcal{S}}^{1}=\widehat{T}_{\mathcal{S}}^{2}$ on $\mathcal{D}_{(-\varrho)}+$ in this case.

For the rest of this section, we fix a good element $\Gamma$ such that

$$
\mathcal{S}=\Gamma+\mathfrak{g}_{x_{0},(-\varrho)^{+}}
$$

with $x_{0} \in \mathcal{B}\left(\mathrm{C}_{\mathrm{G}}(\Gamma), k\right)$. Let $\mathrm{G}^{\prime}:=\mathrm{C}_{\mathrm{G}}(\Gamma)$.

LEMMA 4.2.1. - 1) The invariant distributions $\widehat{\mathrm{T}}_{\mathcal{S}}^{1}$ and $\widehat{\mathrm{T}}_{\mathcal{S}}^{2}$ are elements of $\mathcal{J}_{(-\varrho)^{+}}^{(-\Gamma)}$.

2) The restrictions $\operatorname{res}_{\mathcal{D}_{(-\varrho)}+} \widehat{\mathrm{T}}_{\mathcal{S}}^{1}$ and $\operatorname{res}_{\mathcal{D}_{(-\varrho)}+} \widehat{\mathrm{T}}_{\mathcal{S}}^{2}$ are in the linear span of orbital integrals $\mu_{\mathcal{O}}, \mathcal{O} \in \mathcal{O}(\Gamma)$.

Proof. - Note that 2) is a consequence of 1) and Proposition 4.1.2-(1).

To prove 1), we see by Theorem 4.1.3, 2), we have $\widehat{\mathrm{T}}_{\mathcal{S}}^{2} \in \mathcal{J}_{(-\varrho)^{+}}^{(-\Gamma)}$. To show $\widehat{\mathrm{T}}_{\mathcal{S}}^{1} \in \mathcal{J}_{(-\varrho)^{+}}^{(-\Gamma)}$, let $x \in \mathcal{B}(\mathrm{G}, k)$ and $s \leq(-\varrho)$. We have for $f \in C\left(\mathfrak{g}_{x, s} / \mathfrak{g}_{x,(-\varrho)^{+}}\right)$,

$$
\widehat{\mathrm{T}}_{\mathcal{S}}^{1}(f)=\int_{\mathfrak{g}_{\mathcal{S}}} f(-X) \mathrm{d} X
$$

Then we observe that

(i) $\operatorname{Supp}\left(\widehat{\mathrm{T}}_{\mathcal{S}}^{1}\right)=-\mathfrak{g}_{\mathcal{S}}$;

(ii) if $s<-\varrho, \operatorname{Supp}\left(\widehat{\mathrm{T}}_{\mathcal{S}}^{1}\right)=-\mathfrak{g}_{\mathcal{S}} \subset \mathfrak{g}_{(-\varrho)} \subset \mathcal{N}+\mathfrak{g}_{x, s^{+}}$, by $[2,3.3 .2]$.

If $s=(-\varrho)$, it follows from (i) and the equality $\mathfrak{g}_{\mathcal{S}}={ }^{G}\left(\Gamma+\mathfrak{g}_{(-\varrho)+}^{\prime}\right)$ that $\widehat{\mathrm{T}}_{\mathcal{S}}^{1}$ is in $\mathcal{J}_{x,-\varrho,(-\varrho)^{+}}^{(-\Gamma)}$ If $s<(-\varrho), \widehat{\mathrm{T}}_{\mathcal{S}}^{1} \in \mathcal{J}_{x, s,(-\varrho)^{+}}^{(-\Gamma)}$ is a result of (ii). Hence $\widehat{\mathrm{T}}_{\mathcal{S}}^{1} \in \mathcal{J}_{(-\varrho)^{+}}^{(-\Gamma)}$.

To finish the proof of Theorem 3.3.1, from Proposition 4.1.2, 2), it is enough to check that for $x \in \mathcal{B}\left(\mathrm{G}^{\prime}, k\right)$ and $v \in \mathcal{N}^{\prime} \cap \mathfrak{g}_{x, r}$,

$$
\widehat{\mathrm{T}}_{\mathcal{S}}^{1}\left(\left[-\Gamma+v+\mathfrak{g}_{x,(-\varrho)^{+}}\right]\right)=\widehat{\mathrm{T}}_{\mathcal{S}}^{2}\left(\left[-\Gamma+v+\mathfrak{g}_{x,(-\varrho)^{+}}\right]\right)
$$

Since $\operatorname{Supp}\left(\left[-\Gamma+v+\mathfrak{g}_{x,(-\varrho)^{+}}\right]\right) \subset \mathfrak{g}_{\mathcal{S}}$, we have

$$
\widehat{\mathrm{T}}_{\mathcal{S}}^{1}\left(\left[-\Gamma+v+\mathfrak{g}_{x,(-\varrho)^{+}}\right]\right)=\operatorname{vol}_{\mathfrak{g}}\left(\mathfrak{g}_{x,(-\varrho)^{+}}\right)=\frac{1}{\operatorname{vol}_{\mathfrak{g}}\left(\mathfrak{g}_{x, \varrho}\right)} .
$$

BULLETIN DE LA SOCiÉTÉ MATHÉMATIQUe DE FRANCE 
To compute $\widehat{\mathrm{T}}_{\mathcal{S}}^{2}\left(\left[-\Gamma+v+\mathfrak{g}_{x,(-\varrho)^{+}}\right]\right)$, we find the Fourier transform of $\left[-\Gamma+v+\mathfrak{g}_{x,(-\varrho)^{+}}\right]$. In the following, $f$ denotes $\left[-\Gamma+v+\mathfrak{g}_{x,(-\varrho)^{+}}\right]$.

$$
\begin{aligned}
\widehat{f}(Y) & =\int_{\mathfrak{g}} f(X) \Lambda(\mathrm{B}(X, Y)) \mathrm{d} X=\int_{\mathfrak{g}}\left[\mathfrak{g}_{x,(-\varrho)^{+}}\right](X) \cdot \Lambda(\mathrm{B}(-\Gamma+v+X, Y)) \mathrm{d} X \\
& =\operatorname{vol}_{\mathfrak{g}}\left(\mathfrak{g}_{x,(-\varrho)}\right) \cdot \Lambda(\mathrm{B}(-\Gamma+v, Y)) \cdot\left[\mathfrak{g}_{x, \varrho}\right](Y) \\
& =\frac{1}{\operatorname{vol}_{G}\left(G_{x, \varrho}\right)} \cdot \Lambda(\mathrm{B}(-\Gamma+v, Y)) \cdot\left[\mathfrak{g}_{x, \varrho}\right](Y) .
\end{aligned}
$$

Note that $\operatorname{vol}_{G}\left(G_{x, \varrho}\right) \cdot(\widehat{f} \circ \log )$ is a character of $G_{x, \varrho}$ with dual blob $-\Gamma+v+\mathfrak{g}_{x,(-\varrho)^{+}}$, that is, $\operatorname{vol}_{G}\left(G_{x, \varrho}\right) \cdot(\widehat{f} \circ \log )=\chi_{-\Gamma+v}$. Then $\Theta_{\pi}(\widehat{f} \circ \log )=$ $\Theta_{\pi}\left(\chi_{-\Gamma+v}\right)$ is the multiplicity $m\left(\chi_{\Gamma-v}, V_{\pi}\right)$ of $\chi_{\Gamma-v}$ in $V_{\pi}$. Then by Corollary 3.2.2, any $\left(\pi, V_{\pi}\right)$ with $m\left(\chi_{\Gamma-v}, V_{\pi}\right)>0$ is contained in $\widetilde{G}_{\mathcal{S}}$. Hence $m\left(\chi_{\Gamma-v}, V_{\pi}\right)=0$ unless $\pi \in \widehat{G}_{\mathcal{S}}$, and we have

$$
\begin{aligned}
\frac{1}{\operatorname{vol}_{\mathfrak{g}}\left(\mathfrak{g}_{x, \varrho}\right)} & =\widehat{f}(\log (1))=\int_{\widehat{G}} \Theta_{\pi}(\widehat{f} \circ \log ) \mathrm{d} \pi \\
& =\int_{\widehat{G}_{\mathcal{S}}} \Theta_{\pi}(\widehat{f} \circ \log ) \mathrm{d} \pi=\widehat{\mathrm{T}}_{\mathcal{S}}^{2}\left(\left[-\Gamma+v+\mathfrak{g}_{x,(-\varrho)^{+}}\right]\right) .
\end{aligned}
$$

Now equality (E) follows from (a) and (b). Hence Theorem 3.3.1 is proved when $\varrho>0$.

4.3. Some results on character expansions: depth zero cases. - The following subspaces of $\mathcal{J}(\mathfrak{g})$ are defined in [5, 2.1.1, 2.1.3]. If $r \leq 0$, they characterize the character distributions of irreducible admissible representations of depth $-r$.

Definition 4.3.1. - Let $x \in \mathcal{B}(\mathrm{G}, k)$ and $r \in \mathbb{R}$. For $s<r$, we define $\mathcal{J}_{x, s, r^{+}}^{\Gamma}$ as follows:

$$
\begin{gathered}
\tilde{J}_{x, s, r^{+}}:=\left\{\begin{array}{l|l}
\mathrm{T} \in \mathcal{J}(\mathfrak{g}) & \begin{array}{l}
\text { for } f \in C\left(\mathfrak{g}_{x, s} / \mathfrak{g}_{x, r^{+}}\right) \\
\text {if } \operatorname{Supp}(f) \cap\left(\mathcal{N}+\mathfrak{g}_{x, s^{+}}\right)=\varnothing \\
\operatorname{then} \mathrm{T}(f)=0
\end{array}
\end{array}\right\} . \\
\tilde{J}_{r^{+}}:=\bigcap_{x \in \mathcal{B}(\mathrm{G}, k)} \bigcap_{s \leq r} \tilde{J}_{x, s, r^{+}} .
\end{gathered}
$$

From now on, we fix $\epsilon>0$ such that $\mathfrak{g}_{(-\epsilon)^{+}}=\mathfrak{g}_{0}$ and $\mathcal{D}_{(-\epsilon)^{+}}=\mathcal{D}_{0}$. Such an $\epsilon$ exists. Then we also have $\mathfrak{g}_{\epsilon}=\mathfrak{g}_{0^{+}}$. When $\mathrm{G}$ is a classical group, $\mathfrak{g}_{\epsilon}$ is the set of topologically nilpotent elements.

The following two propositions are corollaries of the results in [18] and [5]. Although their results are more general, we state only the facts necessary to prove Theorem 3.3.1. Note that $\mathcal{O}(0)$ is the set of nilpotent orbits in $\mathfrak{g}$. Denote the span of $\mu_{\mathcal{O}}, \mathcal{O} \in \mathcal{O}(0)$ by $\mathcal{J}_{0}$. 
Proposition 4.3.2. - Suppose $(\mathrm{H} k)$ is valid. Then,

1) $\operatorname{dim}_{\mathbb{C}}\left(\operatorname{res}_{\mathcal{D}_{(-\epsilon)}+} \tilde{J}_{(-\epsilon)+}\right)=|\mathcal{O}(0)|$,

$2) \operatorname{res}_{\mathcal{D}_{(-\epsilon)}+} \tilde{J}_{(-\epsilon)^{+}}=\operatorname{res}_{\mathcal{D}_{(-\epsilon)}+} \mathcal{J}_{0}$

The proposition is from $[5,2.1 .5,3.4 .6]$.

Proposition 4.3.3. - Suppose $(\mathrm{H} k)$ is valid. Let $\left(\pi, V_{\pi}\right)$ be an irreducible admissible representation of $G$ of depth zero. Then,

1) $\widehat{\Theta_{\pi}} \in \tilde{J}_{(-\epsilon)^{+}}$, and

2) the Harish-Chandra-Howe local character expansion of $\Theta_{\pi}$ is valid on $\mathfrak{g}_{(-\epsilon)^{+}}=\mathfrak{g}_{0}$. That is, there are $c_{\mathcal{O}}(\pi) \in \mathbb{C}$ indexed by $\mathcal{O}(0)$, such that for any regular element $X \in \mathfrak{g}_{0^{+}}$,

$$
\Theta_{\pi}(\exp (X))=\sum_{\mathcal{O} \in \mathcal{O}(0)} c_{\mathcal{O}}(\pi) \cdot \widehat{\mu_{\mathcal{O}}}(X) .
$$

These are from $[5,3.3 .2,3.5 .2]$.

Observe that the Fourier transform on $C_{c}^{\infty}(\mathfrak{g})$ maps $C_{c}^{\infty}\left(\mathfrak{g}_{\epsilon}\right)$ to $\mathcal{D}_{(-\epsilon)^{+}}$ (see $[2,4.2 .3]$ ). For the purpose of the proof of Theorem 3.3.1, we introduce Gelfand-Graev functions (see [4]). In [4], although Barbasch and Moy treated only groups considered in [18], since they used only the fact that the HarishChandra-Howe character expansion is valid on $\mathfrak{g}_{0^{+}}$from [18], their results remain valid for any of the reductive groups considered in [5]. Now, we recall some results from [4].

Proposition 4.3 .4 (see $[4,3.7]$ ). - Given a nonzero nilpotent element $N \in \mathfrak{g}$, let $N=e, h, f$ be an $\mathfrak{s l}(2)$-triple associated to $N$. Then there is a point $x \in \mathcal{B}(\mathrm{G}, k)$ so that

1) $e, h, f \in \mathfrak{g}_{x, 0}$, and their images $\bar{e}, \bar{h}, \bar{f}$ in $\mathfrak{m}_{x}:=\mathfrak{g}_{x, 0} / \mathfrak{g}_{x, 0^{+}}$generate an $\mathfrak{s l}(2)$ triple in $\mathfrak{m}_{x}$

2 ) among those parahoric subgroups $G_{x^{\prime}, 0}$ such that $\mathfrak{g}_{x^{\prime}, 0}$ satisfies $(1), G_{x, 0}$ is minimal,

3) if $G_{y, 0}$ is another parahoric subgroup minimal among those parahoric subgroups for which $\mathfrak{g}_{y, 0}$ satisfies (1), then $G_{x, 0}$ and $G_{y, 0}$ are associates.

4.3.5. - For each $\mathcal{O}_{i} \in \mathcal{O}(0)$, fix an element $e_{i} \in \mathcal{O}_{i}$. Choose an $\mathfrak{s l}_{2}$-triple $e_{i}, f_{i}, h_{i}$, and select $x_{i} \in \mathcal{B}(\mathrm{G}, k)$ as in Proposition 4.3.4. Let $f_{x_{i}, \mathcal{O}_{i}}$ be the character of the generalized Gelfand-Graev representation of $M_{x_{i}}:=G_{x_{i}, 0} / G_{x_{i}, 0^{+}}$ associated to $\bar{e}_{i}$ as in $[4,4.5]$. Then $f_{x_{i}, \mathcal{O}_{i}}$ also defines a character on $G_{x_{i}, 0}$ via $G_{x_{i}, 0} \rightarrow M_{x_{i}}$, for which we use the same notation.

Proposition 4.3.6. - We keep the notation from (4.3.5).

1) Each $f_{x_{i}, \mathcal{O}_{i}}$ is supported on the set of unipotent elements in $G_{x_{i}, 0} / G_{x_{i}, 0^{+}}$. Hence it defines a function on $G_{x_{i}, 0}$ supported on the subset $G_{x_{i}, 0} \cap G_{0^{+}}$. 
2) Let $\mathrm{T}_{1}, \mathrm{~T}_{2} \in \mathcal{J}(\mathfrak{g})$. Suppose $\widehat{\mathrm{T}}_{1}, \widehat{\mathrm{T}}_{2} \in \tilde{J}_{(-\epsilon)^{+}}$. Then $\operatorname{res}_{\mathcal{D}_{(-\epsilon)+}} \widehat{\mathrm{T}}_{1}=$ $\operatorname{res}_{\mathcal{D}_{(-\epsilon)}+} \widehat{T}_{2}$ if and only if $\mathrm{T}_{1}\left(f_{x_{i}, \mathcal{O}_{i}} \circ \exp \right)=\mathrm{T}_{2}\left(f_{x_{i}, \mathcal{O}_{i}} \circ \exp \right), \mathcal{O}_{i} \in \mathcal{O}(0)$.

Part 1) is [4, 4.5]. Part 2) follows from Proposition 4.3.2 and [4, 4.7].

We remark that $f_{x_{i}, \mathcal{O}_{i}} \circ \exp$ separate the nilpotent orbital integrals in $\mathfrak{g}[4]$.

4.4. Proof of Theorem 3.3.1: the depth zero case. - We keep the notation from 4.3. Fix a 0-good coset $\mathcal{S}$. Then $\mathfrak{g}_{\mathcal{S}}=\mathfrak{g}_{0}$. Recall $\mathfrak{g}_{\epsilon}=\mathfrak{g}_{0+}$ and $\mathcal{D}_{(-\epsilon)^{+}}=\sum_{x \in \mathcal{B}(\mathrm{G}, k)} C_{c}\left(\mathfrak{g} / \mathfrak{g}_{x,(-\epsilon)^{+}}\right)=\sum_{x \in \mathcal{B}(\mathrm{G}, k)} C_{c}\left(\mathfrak{g} / \mathfrak{g}_{x, 0}\right)$. Then the Fourier transform on $C_{c}^{\infty}(\mathfrak{g})$ maps $C_{c}^{\infty}\left(\mathfrak{g}_{\epsilon}\right)$ to $\mathcal{D}_{(-\epsilon)^{+}}($see $[2,4.2 .3])$.

Let $\mathrm{T}_{0}^{1}$ and $\mathrm{T}_{0}^{2}$ be invariant distributions defined as follows: for $f \in C_{c}^{\infty}(\mathfrak{g})$, recall $f_{0^{+}}=f \cdot\left[\mathfrak{g}_{0^{+}}\right]$. Then

$$
\begin{aligned}
& \mathrm{T}_{0}^{1}(f):=\int_{\mathfrak{g}_{0}} \widehat{f_{0^{+}}}(X) \mathrm{d} X, \\
& \mathrm{~T}_{0}^{2}(f):=\int_{\widehat{G}_{0}} \widehat{f_{0^{+}} \circ \log }(\pi) \mathrm{d} \pi=\int_{\widehat{G}_{0}} \Theta_{\pi}\left(f_{0^{+}} \circ \log \right) \mathrm{d} \pi .
\end{aligned}
$$

Then to prove the theorem, it is enough to prove that $\mathrm{T}_{0}^{1}(f)=\mathrm{T}_{0}^{2}(f)$ for any $f \in C_{c}^{\infty}\left(\mathfrak{g}_{\epsilon}\right)$. Contrary to the positive depth cases, we will directly prove that $\mathrm{T}_{0}^{1}=\mathrm{T}_{0}^{2}$ on $C_{c}^{\infty}\left(\mathfrak{g}_{\epsilon}\right)$. We use Gelfand-Graev functions $f_{x, \mathcal{O}}$ as test functions.

Lemma 4.4.1. - The restrictions $\operatorname{res}_{\mathcal{D}_{(-\epsilon)+}} \widehat{T}_{0}^{1}$ and $\operatorname{res}_{\mathcal{D}_{(-\epsilon)}+} \widehat{T}_{0}^{2}$ are in the linear span of orbital integrals $\operatorname{res}_{\mathcal{D}_{(-\epsilon)+}+} \mu_{\mathcal{O}}, \mathcal{O} \in \mathcal{O}(0)$. Moreover, on $C_{c}^{\infty}\left(\mathfrak{g}_{\epsilon}\right), \mathrm{T}_{0}^{1}$ and $\mathrm{T}_{0}^{2}$ are in the linear span of $\widehat{\mu_{\mathcal{O}}}, \mathcal{O} \in \mathcal{O}(0)$.

Proof. - The second statement is a consequence of the first one via Fourier transform. The first statement for $\widehat{\mathrm{T}}_{0}^{2}$ follows from Proposition 4.3.3. To prove it for $\widehat{\mathrm{T}}_{0}^{1}$, we observe that $\operatorname{Supp}\left(\widehat{\mathrm{T}}_{0}^{1}\right)=-\mathfrak{g}_{0} \subset \mathfrak{g}_{0} \subset \mathcal{N}+\mathfrak{g}_{x, s^{+}}$for any $s \leq-\epsilon$ $[2,3.2 .2]$. Then $\widehat{T}_{0}^{1} \in \tilde{J}_{(-\epsilon)^{+}}$from the definition of $\tilde{J}_{x, s,(-\epsilon)^{+}}$and $\tilde{J}_{(-\epsilon)^{+}}$.

By Proposition 4.3.6, it is now enough to check that

$$
\mathrm{T}_{0}^{1}\left(f_{x, \mathcal{O}} \circ \exp \right)=\mathrm{T}_{0}^{2}\left(f_{x, \mathcal{O}} \circ \exp \right)
$$

for each $(x, \mathcal{O}):=\left(x_{i}, \mathcal{O}_{i}\right)$ in (4.3.5).

Since $\Theta_{\pi}\left(f_{x, \mathcal{O}}\right) \neq 0$ implies that $\pi$ has depth zero, we have

$$
f_{x, \mathcal{O}}(1)=\int_{\widehat{G}} \Theta_{\pi}\left(f_{x, \mathcal{O}}\right) \mathrm{d} \pi=\int_{\widehat{G}_{\mathcal{S}}} \Theta_{\pi}\left(f_{x, \mathcal{O}}\right) \mathrm{d} \pi=\mathrm{T}_{0}^{2}\left(f_{x, \mathcal{O}} \circ \exp \right) .
$$

Denote $f_{x, \mathcal{O}} \circ \exp$ by $\gamma_{N}$. Since $\operatorname{Supp}\left(\gamma_{N}\right) \subset \mathfrak{g}_{0^{+}}, \operatorname{Supp}\left(\widehat{\gamma}_{N}\right) \subset \mathfrak{g}_{0}$. Then

$\left(\mathrm{b}_{0}\right) \quad \mathrm{T}_{0}^{1}\left(f_{x, \mathcal{O}} \circ \exp \right)=\int_{\mathfrak{g}_{0}} \widehat{\gamma}_{N}(X) \mathrm{d} X=\int_{\mathfrak{g}} \widehat{\gamma}_{N}(X) \mathrm{d} X=\gamma_{N}(0)=f_{x, \mathcal{O}}(1)$.

TOME $132-2004-\mathrm{N}^{\mathrm{O}} 1$ 
Hence the equality $\left(\mathrm{E}_{0}\right)$ follows from $\left(\mathrm{a}_{0}\right),\left(\mathrm{b}_{0}\right)$, and Theorem 3.3.1 is proved.

4.5. Application. - In this section, we prove that every irreducible tempered representation contains a good minimal K-type, when hypothesis $(\mathrm{H} k)$ is valid. We apply Theorem 3.3.1 for the main step of the proof. We also give another proof for the fact that any irreducible admissible representation contains a good minimal K-type (see $[13,2.4 .10]$ ) under the same hypothesis. This is a weaker result due to a stronger assumption, and there might be simpler ways to prove this. However, in approaching the problem of the exhaustion of types constructed in [12] or supercuspidal representations in [20], we believe this approach will be useful. As a corollary, we get a spectral decomposition of the delta distribution on $G$.

THEOREM 4.5.1. - Assume (Hk) and (HGT) are valid.

1) Any irreducible tempered representation contains a good minimal K-type.

2) Any irreducible admissible representation contains a good minimal K-type, that is, $\widetilde{G}=\bigcup_{\mathcal{S} \in \mathfrak{S}} \widetilde{G}_{\mathcal{S}}$.

Proof. - 1) We fix $x_{0} \in \mathcal{B}(\mathrm{G}, k)$ and let $s \in \mathbb{R}_{\geq 0}$. For simplicity, we denote $G_{x_{0}, s^{+}}$by $K_{s}$. Let $f_{s}$ be $\left[K_{s}\right]$, the characteristic function supported on $K_{s}$. Let

$$
\widehat{G}^{K_{s}}:=\left\{\left(\pi, V_{\pi}\right) \in \widehat{G} \mid V_{\pi}^{K_{s}} \neq 0\right\} \quad \text { and } \quad \widehat{G}_{\mathcal{S}}^{K_{s}}:=\widehat{G}_{\mathcal{S}} \cap \widehat{G}^{K_{s}} \text {. }
$$

Clearly, $\bigcup_{\mathcal{S} \in \mathfrak{S}} \widehat{G}_{\mathcal{S}} \subset \widehat{G}$. To prove that $\widehat{G} \subset \bigcup_{\mathcal{S} \in \mathfrak{S}} \widehat{G}_{\mathcal{S}}$, we compute

$$
\begin{aligned}
\int_{\mathfrak{g}} \widehat{f_{s} \circ \exp }(X) \mathrm{d} X & =\int_{\widehat{G}} \widehat{f}(\pi) \mathrm{d} \pi \stackrel{(\mathrm{i})}{\geq} \sum_{\mathcal{S} \in \mathfrak{S}} \int_{\widehat{G}_{\mathcal{S}}^{K_{s}}} \widehat{f}(\pi) \mathrm{d} \pi \\
& =\sum_{\substack{\mathcal{S} \in \mathfrak{S} \\
\widehat{G}_{\mathcal{S}}^{K} \neq \varnothing}} \int_{\widehat{G}_{\mathcal{S}}^{K_{s}}} \widehat{f}(\pi) \mathrm{d} \pi \stackrel{(\mathrm{ii})}{=} \sum_{\substack{\mathcal{S} \in \mathfrak{S} \\
\widehat{G}_{\mathcal{S}}^{K} \neq \varnothing}} \int_{\mathfrak{g}_{\mathcal{S}}} \widehat{f_{s} \circ \exp }(X) \mathrm{d} X \\
& \stackrel{(\mathrm{iii})}{=} \sum_{\mathcal{S} \in \mathfrak{S}} \int_{\mathfrak{g}_{\mathcal{S}}} \widehat{f_{s} \circ \exp }(X) \mathrm{d} X \stackrel{(\mathrm{iv})}{=} \int_{\mathfrak{g}} \widehat{f_{s} \circ \exp }(X) \mathrm{d} X .
\end{aligned}
$$

Relations (i), (iii) and (iv) are rather straightforward. We use Theorem 3.3.1 to verify (ii).

The first inequality (i) follows from $\bigcup_{\mathcal{S} \in \mathfrak{S}} \widehat{G}_{\mathcal{S}} \subset \widehat{G}$. To prove (ii), it is enough to verify that for $\mathcal{S} \in \mathfrak{S}$, if $\widehat{G}_{\mathcal{S}}^{K_{s}} \neq \varnothing$,

$$
\int_{\widehat{G}_{\mathcal{S}}^{K_{s}}} \widehat{f}(\pi) \mathrm{d} \pi=\int_{\mathfrak{g}_{\mathcal{S}}} \widehat{f_{s} \circ \exp }(X) \mathrm{d} X .
$$

Since $f_{s}(\pi) \neq 0$ only if $s \geq \varrho(\pi)$, and since $\mathrm{d}(\mathcal{S})=\varrho(\pi)$ for any $\pi \in \widehat{G}_{\mathcal{S}}$, we see that $\widehat{G}_{\mathcal{S}}^{K_{s}} \neq \varnothing$ only if $s \geq \mathrm{d}(\mathcal{S})$. In that case, since $f_{s} \circ \exp \in C_{c}^{\infty}\left(\mathfrak{g}_{\mathrm{d}(\mathcal{S})^{+}}\right) \subset$ 
$C_{c}^{\infty}\left(\mathfrak{g}_{\mathrm{d}(\mathcal{S})}\right)$, the equality in Theorem 3.3.1 is valid for $f_{s}$. Hence the above equality is valid, and so is (ii). To prove (iii), we note that $\widehat{f_{s} \circ \exp }$ is a scalar multiple of the characteristic function of $\mathfrak{g}_{x_{0},-s}$. Then since

$$
\operatorname{Supp}\left(\widehat{f_{s} \circ \exp }\right) \cap \mathfrak{g}_{\mathcal{S}}=\mathfrak{g}_{x_{0},-s} \cap \mathfrak{g}_{\mathcal{S}} \neq \varnothing
$$

only if $\widehat{G}_{\mathcal{S}}^{K_{s}} \neq \varnothing$, equality (iii) holds. From Lemma 1.4 .5 , (iv) is valid. Hence ' $\geq$ ' in (i) is in fact an equality for all $s \in \mathbb{R}_{\geq 0}$. Therefore, $\widehat{G}^{K_{s}}=\bigcup_{\mathcal{S} \in \mathfrak{S}} \widehat{G}_{\mathcal{S}}^{K_{s}}$. Since $\widehat{G}=\bigcup_{s \in \mathbb{R}_{\geq 0}} \widehat{G}^{K_{s}}$ and $\widehat{G}_{\mathcal{S}}=\bigcup_{s \in \mathbb{R}_{\geq 0}} \widehat{G}_{\mathcal{S}}^{K_{s}}$, we can now conclude $\widehat{G}=\bigcup_{\mathcal{S} \in \mathfrak{S}} \widehat{G}_{\mathcal{S}}$.

2) We prove this in two ways.

Proof 1. - We observe that for any minimal K-type $\mathfrak{s}=\left(G_{x, \varrho}, \chi\right)$, we have $\widetilde{G}_{\mathfrak{s}} \cap \widehat{G} \neq \varnothing$. This follows from the Plancherel formula on the group side:

$$
\chi(1)=\int_{\widehat{G}} m(\chi, \pi) \mathrm{d} \pi=\int_{\widehat{G}_{\mathfrak{s}}} m(\chi, \pi) \mathrm{d} \pi>0 .
$$

Hence, there is $\mathcal{S} \in \mathfrak{S}$ such that $\widetilde{G}_{\mathfrak{s}} \cap \widetilde{G}_{\mathcal{S}} \neq \varnothing$.

Now it is enough to prove that if $\widetilde{G}_{\mathcal{S}} \cap \widetilde{G}_{\mathfrak{s}} \neq \varnothing$, then $\widetilde{G}_{\mathcal{S}}=\widetilde{G}_{\mathfrak{s}}$.

From the definition of weak associativities, we have $\widetilde{G}_{\mathcal{S}} \subset \widetilde{G}_{\mathfrak{s}}$. Now, it is enough to show $\widetilde{G}_{\mathfrak{s}} \subset \widetilde{G}_{\mathcal{S}}$. Let $\left(\pi, V_{\pi}\right) \in \widetilde{G}_{\mathfrak{s}}$. Without loss of generality, we may assume that $V_{\pi}$ contains $\mathfrak{s}$. Write $\mathcal{S}=\Gamma+\mathfrak{g}_{x,(-\varrho)^{+}}$. Note that there is a sequence of nondegenerate dual cosets $\mathcal{S}=\mathcal{Y}_{0}, \mathcal{Y}_{1}, \cdots, \mathcal{Y}_{k}=\mathfrak{s}^{\sharp}$ of the form $\mathcal{Y}_{i}=Y_{i}+\mathfrak{g}_{x_{i},(-\varrho)} \subset \subset \mathfrak{g}_{x_{i},(-\varrho)}$, such that $\mathcal{Y}_{i} \cap{ }^{g_{i}} \mathcal{Y}_{i+1} \neq \varnothing$ for some $g_{i} \in G$. By Lemma 1.3.4, there are $y_{i} \in \mathcal{B}\left(\mathrm{C}_{\mathrm{G}}(\Gamma), k\right), n_{i}^{\prime} \in \mathcal{N} \cap \mathfrak{g}^{\prime}$ and $h_{i} \in G$ such that $\mathcal{Y}_{i}={ }^{h_{i}}\left(\Gamma+n_{i}^{\prime}+\mathfrak{g}_{y_{i},(-\varrho)^{+}}\right)$. In particular, $\mathfrak{s}^{\sharp}={ }^{h_{k}}\left(\Gamma+n_{k}^{\prime}+\mathfrak{g}_{y_{k},(-\varrho)^{+}}\right)$. Then by Corollary $3.2 .2, \mathfrak{s}$ contains a good minimal K-type weakly associated to $\mathcal{S}$.

Proof 2. - Let $\left(\pi, V_{\pi}\right) \in \widetilde{G}$. Let $(M, \sigma)$ be a cuspidal support of $\left(\pi, V_{\pi}\right)$, which consists of a Levi subgroup $M$ of $G$ and a supercuspidal representation $\sigma$, such that $\left(\pi, V_{\pi}\right)$ occurs as a subquotient of $\operatorname{Ind}_{M}^{G}(\sigma)$. Since $\sigma$ is essentially tempered (that is, there is an unramified character $\xi$ of $M$ such that $\sigma \otimes \xi$ is tempered), $\sigma$ contains a good minimal K-type by $(1)$. Let $\left(M_{x, \rho}, \chi_{\gamma}\right)$ be a good minimal K-type contained in $\left(\sigma, V_{\sigma}\right)$, and let $\gamma+\mathfrak{m}_{x,(-\varrho)}+$ be its good dual blob. Choose a maximal $k$-torus $\mathrm{T} \subset \mathrm{M}$ which splits over a tamely ramified extension such that $\gamma \in \mathfrak{t}$. Since $\mathrm{T}$ is also a maximal $k$-torus, by (HGT), there is a good element $\Gamma \in \gamma+\mathfrak{t}_{(-\varrho)^{+}}$in $\mathfrak{g}$ such that $\gamma+\mathfrak{t}_{(-\varrho)^{+}}=\Gamma+\mathfrak{t}_{(-\varrho)^{+}}$. By Lemma 1.3.5, we have $\gamma+\mathfrak{m}_{x,(-\varrho)^{+}}=\Gamma+\mathfrak{m}_{x,(-\varrho)^{+}}$, which implies $\left(M_{x, \varrho}, \chi_{\gamma}\right)=\left(M_{x, \varrho}, \chi_{\Gamma}\right)$. By Remark 1.3.3, 3), $x \in \mathcal{B}\left(\mathrm{C}_{\mathrm{M}}(\Gamma), k\right) \subset \mathcal{B}\left(\mathrm{C}_{\mathrm{G}}(\Gamma), k\right)$. Hence $\Gamma+\mathfrak{g}_{x,(-\varrho)^{+}}$is a good coset. Now since $\sigma$ contains $\left(M_{x, \varrho}, \chi_{\Gamma}\right)$, by $[15,5.2]$, any subquotient of $\operatorname{Ind}_{M}^{G} \sigma$, in particular $\left(\pi, V_{\pi}\right)$, contains a good type $\mathfrak{s}=\left(G_{x, \varrho}, \chi_{\Gamma}\right)$.

TOME $132-2004-\mathrm{N}^{\mathrm{O}} 1$ 
Corollary 4.5.2. - Assume $(\mathrm{H} k)$ and (HGT) are valid. For any $f \in C_{c}^{\infty}(G)$, we have

$$
f(1)=\sum_{\mathcal{S} \in \mathfrak{S}} \int_{\widehat{G}_{\mathcal{S}}} \Theta_{\pi}(f) \mathrm{d} \pi .
$$

Proof. - This follows from Theorem 4.5.1 and $\widehat{G}=\bigcup_{\mathcal{S} \in \mathfrak{S}} \widehat{G}_{\mathcal{S}}$.

\section{BIBLIOGRAPHY}

[1] AdLer (J.) - Refined anisotropic k-types and supercuspidal representations, Pacific J. Math., t. 185 (1998), pp. 1-32.

[2] Adler (J.) \& DeBACKer (S.) - Some applications of Bruhat-Tits theory to harmonic analysis on the Lie algebra of a reductive p-adic group, Michigan J. Math., t. 50 (2002), no. 2, pp. 263-286.

[3] Adler (J.) \& Roche (A.) - An intertwining result for p-adic groups, Canad. J. Math., t. 52 (2000), no. 3, pp. 449-467.

[4] Barbasch (D.) \& Moy (A.) - Local character expansions, Ann. Sci. École Norm. Sup., t. 30 (1997), no. 5, pp. 553-567.

[5] DeBACKer (S.) - Homogeneity results for invariant distributions of a reductive p-adic group, Ann. Sci. École Norm. Sup., t. 35 (2002), pp. 391422.

[6] _ Parameterizing nilpotent orbits via Bruhat-Tits theory, Ann. of Math., t. 156 (2002), pp. 295-331.

[7] Dixmier (J.) - Les $C^{*}$-algèbres et leurs représentations, Éditions Jacques Gabay, Paris, 1996.

[8] HARISH-ChANDRA - The Plancherel formula for reductive p-adic groups, in Collected Papers, vol. 4, Springer-Verlag, Berlin, 1976.

[9] _ Admissible invariant distributions on reductive p-adic groups, University Lecture Series, vol. 16, Amer. Math. Soc., Providence, RI, 1999, Preface and notes by Stephen DeBacker and Paul J. Sally, Jr.

[10] Howe (R.) - Kirillov theory for compact p-adic groups, Pacific J. Math., t. 73 (1977), pp. 365-381.

[11] _ Some qualitative results on the representation theory or $\mathrm{Gl}_{n}$ over a p-adic field, Pacific J. Math., t. 73 (1977), pp. 479-538.

[12] KIM (J.L.) - Hecke algebras of classical groups over p-adic fields and supercuspidal representations, Amer. J. Math., t. 121 (1999), pp. 967-1029.

[13] Kim (J.L.) \& Murnaghan (F.) - Character expansions and unrefined minimal K-types, Preprint, 2002.

[14] Moy (A.) \& PRAsad (G.) - Unrefined minimal K-types for p-adic groups, Invent. Math., t. 116 (1994), pp. 393-408.

BULLETIN DE LA SOCIÉtÉ MATHÉMATIQUE DE FRANCE 
[15] - Jacquet functors and unrefined minimal K-types, Comment. Math. Helv., t. 71 (1996), pp. 98-121.

[16] Prasad (G.) - Galois fixed points in the Bruhat-Tits buildings of a reductive group, Bull. Soc. Math. France, t. 129 (2001), pp. 169-174.

[17] Rousseau (G.) - Immeubles des groupes réductifs sur les corps locaux, Ph.D. Thesis, Paris XI, 1977.

[18] Waldspurger (J.-L.) - Homogénéité de certaines distributions sur les groupes p-adiques, Inst. Hautes Études Sci. Publ. Math., t. 81 (1995), pp. 25-72.

[19] _ La formule de Plancherel pour les groupes p-adiques d'après Harish-Chandra, J. Inst. Math. Jussieu, t. 2 (2003), no. 2, pp. 235-333.

[20] YU (J.K.) - Construction of tame supercuspidal representations, J. Amer. Math. Soc., t. 14 (2001), no. 3, pp. 579-622. 